HYDROLOGIC, METEOROLOGICAL, AND UNSATURATED-ZONE

MOISTURE-CONTENT DATA, FRANKLIN LAKE PLAYA,

INYO COUNTY, CALIFORNIA

By John B. Czarnecki

U.S. GEOLOGICAL SURVEY

Open-File Report 89-595

Prepared in cooperation with the

NEVADA OPERATIONS OFFICE,

U.S. DEPARTMENT OF ENERGY, under

Interagency Agreement DE-AI08-78ET44802

Denver, Colorado

1990 
DEPARTMENT OF THE INTERIOR

MANUAL LUJAN, JR., Secretary

U.S. GEOLOGICAL SURVEY

Dallas L. Peck, Director

For additional information write to:

Chief, Nuclear Hydrology Program U.S. Geological Survey Water Resources Division Box 25046, Mail Stop 421 Denver Federal Center Lakewood, CO 80225-0046
Copies of this report can be purchased from:

U.S. Geological Survey Books and Open-File Reports Section Federal Center

Box 25425

Denver, CO 80225-0425

[Telephone: (303) 236-7476] 


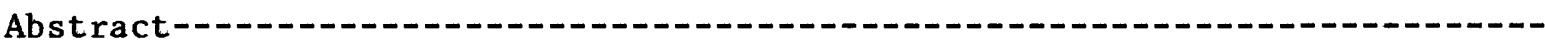

Introduction-1-0-

Purpose and scope-10

Previous work-

Acknowledgments--

Hydrologic data--

Hydraulic head--

Vertical hydraulic gradients--

Meteorological data-- 18

Moisture content in the unsaturated zone-1

Summary---no

References cited-- 39

\section{FIGURES}

Figure 1. Map showing location of the study area- Page

2. Map showing study sites at Franklin Lake playa--- 5

$3 A-H$. Hydrographs showing water-level altitude in:

$3 A$. Wells 1,3 , and 5

$3 B$. Wells 6,7 , and 8

3C. Wells $10,11,13$, and 14

3D. Wells GS-1, GS-2, GS-3, GS-4, GS-5, GS-6, and GS-7------ 7

$3 E$. Wells GS-9, GS-10, and GS-11- 8

$3 F$. Wells GS-12, GS-13, and GS-14- 8

3G. We1ls GS-15, GS-16, and GS-17- 9

3H. Wells GS-18, GS-19, and GS-20 9

4. Graph showing example of line of best fit through waterlevel-altitude data versus well-depth data obtained on May 18, 1983, for wells 1,3 , and 5- 10

$5 A-G$. Graphs showing vertical hydraulic gradient and regression coefficient for:

5A. Wells 1,3 , and $5-11$

$5 B$. Wells 6,7 , and 8

5C. Wells 4, 10, 11 , and GS-20-_ 13

5D. Wells GS-3, GS-4, GS-5, and GS-7-- 14

5E. Wells GS-9, GS-10, and GS-11- 15

$5 F$. Wells GS-15, GS-16, and GS-17- 16

5G. Wells GS-13, GS-14, and GS-20-17

$\begin{aligned} 6 A-F & \text { Graphs showing moisture-content profiles for: } \\ 6 \mathrm{~A} \text {. Well GS-4- } & \end{aligned}$

6B. Well GS-5- 22

6C. Well GS-6- 23

6D. Wel1 GS-15-- 23

6E. We11 GS-18- 24

6F. Well GS-20-14

$7 A-F$. Graphs showing relation between moisture flux and the difference in time between measurements for:

7A. Well GS-4- 27

7B. Well GS-5

7C. Well GS-6- 31 
Figures $7 A-F$. Graphs showing relation between moisture flux and the difference in time between measurements for--Continued:

7D. We11 GS-15- 31

7E. Well GS-18- 35

7F. We11 GS-20 35

8. Histogram showing distribution of fluxes estimated from changes in soil-moisture contents with time-- 36

\section{TABLES}

Table 1. Climatological summary for June 1978 to May 1983 for

Camp Desert Rock, Mercury, Nevada-19

2. Summary of temperatures, wind speeds, and pan-evaporation rates for Boulder City, Nevada, 1982-83- 20

3. Summary of temperatures, wind speeds, and pan-evaporation rates for Silverpeak, Nevada, 1982-83- 21

$4 A-F$. Summary of flux values obtained from different neutron logs of we11:

4A. GS-4- 26

4B. GS-5- 27

4C. GS-6-15 29

4D. GS-15- 30

4E. GS-18- 33

4F. GS-20 34

\section{CONVERSION FACTORS}

\section{Multiply}

centimeter per month

centimeter per day

kilometer per hour

kilometer per day

meter $(\mathrm{m})$

meter per annum (m/a)

millibars

millimeter per month

foot

inch

mile per hour

mile per day

mile per month

inch per month

inch per day
By

0.3937

0.3937

0.621

0.0259

3.281

3.281

0.000987

0.3937

30.48

2.54

1.609

1.609

1.609

2.54

2.54
To obtain

inch per month

inch per day

mile per hour

mile per hour

foot

foot per year

atmosphere

inch per month

centimeter

centimeter

kilometer per hour

kilometer per day

kilometer per month

centimeter per month

centimeter per day

Temperature can be converted to degree Celsius $\left({ }^{\circ} \mathrm{C}\right)$ or degree Fahrenheit $\left({ }^{\circ} \mathrm{F}\right)$ by the equations:

$$
\begin{aligned}
& { }^{\circ} \mathrm{C}=5 / 9\left({ }^{\circ} \mathrm{F}-32\right) \\
& { }^{\circ} \mathrm{F}=9 / 5\left({ }^{\circ} \mathrm{C}\right)+32
\end{aligned}
$$




\title{
HYDROLOGIC, METEOROLOGICAL, AND UNSATURATED-ZONE MOISTURE-CONTENT DATA, FRANKLIN LAKE PLAYA, INYO COUNTY, CALIFORNIA
}

By John B. Czarnecki

\begin{abstract}
Hydrologic and other data were collected at Franklin Lake playa, one of the principal discharge areas of the Furnace Creek Ranch-Alkali Flat groundwater flow system, located in southern Nevada and adjacent California. This data will be used to further characterize ground-water discharge that occurs at the playa largely as bare-soil evaporation. Data presented include:

(1) Hydrographs of water levels in piezometers; (2) vertical hydraulic gradients estimated from piezometer-nest data; (3) meteorological data from weather stations in the vicinity of Franklin Lake playa; and (4) estimates of moisture fluxes based on changes in soil-moisture content in the unsaturated zone.
\end{abstract}

\section{INTRODUCTION}

In arid-climate, regional ground-water-flow systems, discharge that results from evapotranspiration is a major component of ground-water flux. Accurate determination of the rate of evapotranspiration is needed to estimate ground-water flow rates and directions within these systems because evapotranspiration can be an important boundary condition in simulations of the flow system. Further, evapotranspiration generally can be measured more easily than its counterpart, recharge, and for this reason typically is specified explicitly and plays a significant role in the mass balance of these models.

Yucca Mountain (fig. 1), located on the western edge of the Nevada Test Site, is being studied by the U.S. Department of Energy as a potential site for a mined geologic repository for storing high-level radioactive waste. As part of these studies, the U.S. Geological Survey has been investigating the ground-water flow system beneath Yucca Mountain and vicinity because of the potential for ground water to transport radionuclides away from a repository. These investigations, done in cooperation with the U.S. Department of Energy under Interagency Agreement DE-AI08-78ET44802, are part of the Nevada Nuclear Waste Storage Investigations.

The ground-water flow system beneath Yucca Mountain and vicinity was studied and modeled (Czarnecki and Waddell, 1984) using a parameter-estimation method to provide an understanding of the ground-water flow system and of the sensitivity of the model to changes in model-flux variables. From the sensitivity analyses performed, the ground-water discharge that occurs as 


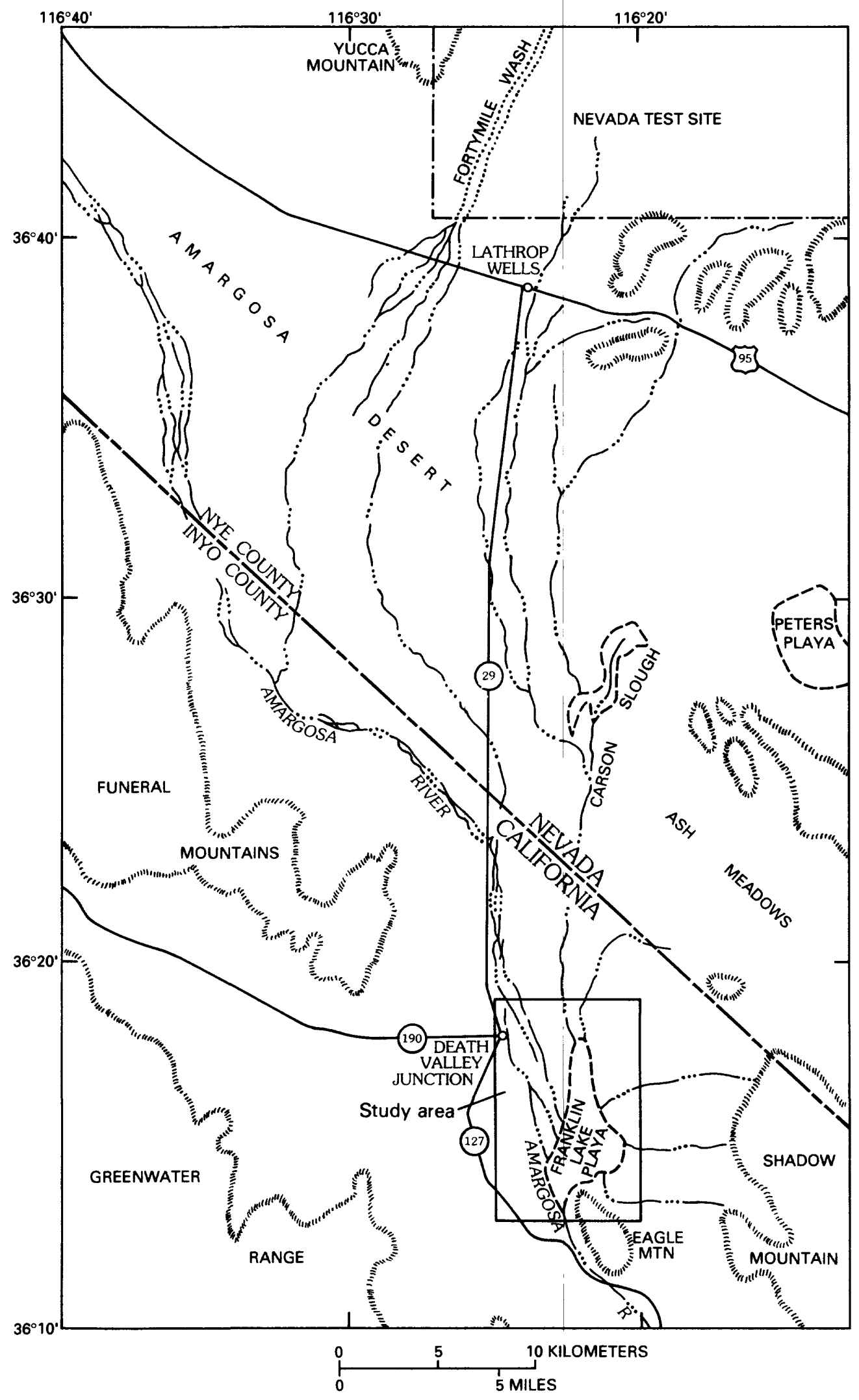

Figure 1.--Location of the study area. 
evapotranspiration at Franklin Lake playa, one of the principal ground-waterdischarge areas, was determined to have the largest effect on the calculation of transmissivity values at, and downgradient from, Yucca Mountain. Because little was known about the rate of ground-water discharge and evapotranspiration, onsite investigations were made at Franklin Lake playa to quantify these rates and to determine the position of the water table and values of other hydrologic variables. Onsite investigations began in May 1983 and continued until July 1985 . This report documents the findings made during this period.

\section{Purpose and Scope}

The purpose of this report is to present data pertinent to the characterization of the geohydrology and evapotranspiration at Franklin Lake playa. These data are analyzed (Czarnecki, 1987) using a variety of methods to estimate evapotranspiration at Franklin Lake playa. The scope of this report is limited to reporting only certain data used for characterization; no interpretation is contained herein.

\section{Previous Work}

In their study of the hydrology of the Amargosa Desert, Walker and Eakin (1963, p. 23) provided an approximate estimate of the rate of evaporation at Franklin Lake playa (known also as Alkali Flat). Their estimated evaporation rate of $0.3 \mathrm{~m} / \mathrm{a}$ was based in part on recharge estimates for the Amargosa Desert, using an empirical procedure developed by Eakin and others (1951). Later work was performed by Calzia and others (1979) in which a single drill hole was drilled near the center of the playa by a reversecirculation process and drill cuttings were logged. The purpose of this hole was, in part, to characterize the mineral potential of the playa waters and sediments, particularly for lithium content. These cuttings later were analyzed by Pantea (1980).

Several wells were drilled at the southern end of the playa (Fred Johnson, American Borate Co., oral commun., 1983) for the purpose of recovering gold and silver thought to be dissolved in the playa waters. At least 12 holes were drilled between 1978 and 1980, ranging in depth from 3 to $17 \mathrm{~m}$. No gold or silver ever was found. However, some of these wells were used in this study to obtain hydrologic and hydrochemical data.

Regional analyses of the ground-water flow system that includes Franklin Lake were conducted by Rush (1970), Winograd and Thordarson (1975), Waddell (1982), Czarnecki and Waddel1 (1984) and Czarnecki (1985). However, none of these efforts involved direct measurements of hydrologic properties at Franklin Lake playa.

\section{Acknowledgments}

The author is indebted to several field assistants from the U.S. Geological Survey for the numerous long, arduous trips made to collect water samples and water-level, moisture-content, and surveying data. This group of people includes William Oatfield, William Townsend, and Ronald Spaulding. 


\section{HYDROLOGIC DATA}

Characterization of the geohydrology at Franklin Lake playa includes the determination of the potentiometric surface using a network of piezometers and unused wells that are located throughout the study area. Data in the following sections were interpreted by Czarnecki (1987) as part of characterization of the geohydrology of the playa. Piezometers and wells also were used for hydraulic testing to determine transmissivity and hydraulicconductivity values. These results also were interpreted by Czarnecki (1987).

\section{Hydraulic Head}

Hydraulic-head data were collected from a network of 20 piezometers (GS-1 through GS-20, fig. 2) installed on or near the playa and from 15 unused wells on or near the playa. Wells GS-1 through GS-20 were installed in nests at different locations to obtain hydraulic-head measurements at various depths below land surface at a specified site.

A11 piezometers constructed during this study were designated with a "GS" prefix and were numbered consecutively north to south and east to west. Well numbers not preceded by a prefix represent wells that existed prior to this study and were numbered in the order in which they were found. Missing well numbers $(2,4,9$, and 12) represent uncased boreholes that were found at Franklin Lake playa but were not used in this study because of problems with surface-water inflows resulting from infrequent, major storms.

Water levels in numerous wells were measured. These levels are shown in figures $3 A-H$. Measurements were made using a steel tape; measurement. precision generally is to within $0.002 \mathrm{~m}$. Flat hydrographs are typical of wells completed in transmissive sediments; hydrographs with fluctuations indicate less permeable sediments.

\section{Vertical Hydraulic Gradients}

Vertical hydraulic gradients were estimated from potentiometric data obtained at various piezometer nests at Franklin Lake playa. An example graph of the line of best fit through water-level altitude data versus welldepth data obtained on May 18, 1983, for wells 1, 3, and 5 is shown in figure 4. The slope of the line of best fit $(0.253)$ is an approximate value of the vertical hydraulic gradient (or change in hydraulic head with depth). Positive gradient values indicate increasing hydraulic head with increasing depth as would be expected in a discharge area. These estimates are shown in figures $5 A-G$ and are based on the slope of the line of best fit through plots showing the relation between hydraulic head and piezometer depth. The regression coefficient for each gradient calculation (slope of the line of best fit) has also been plotted. 


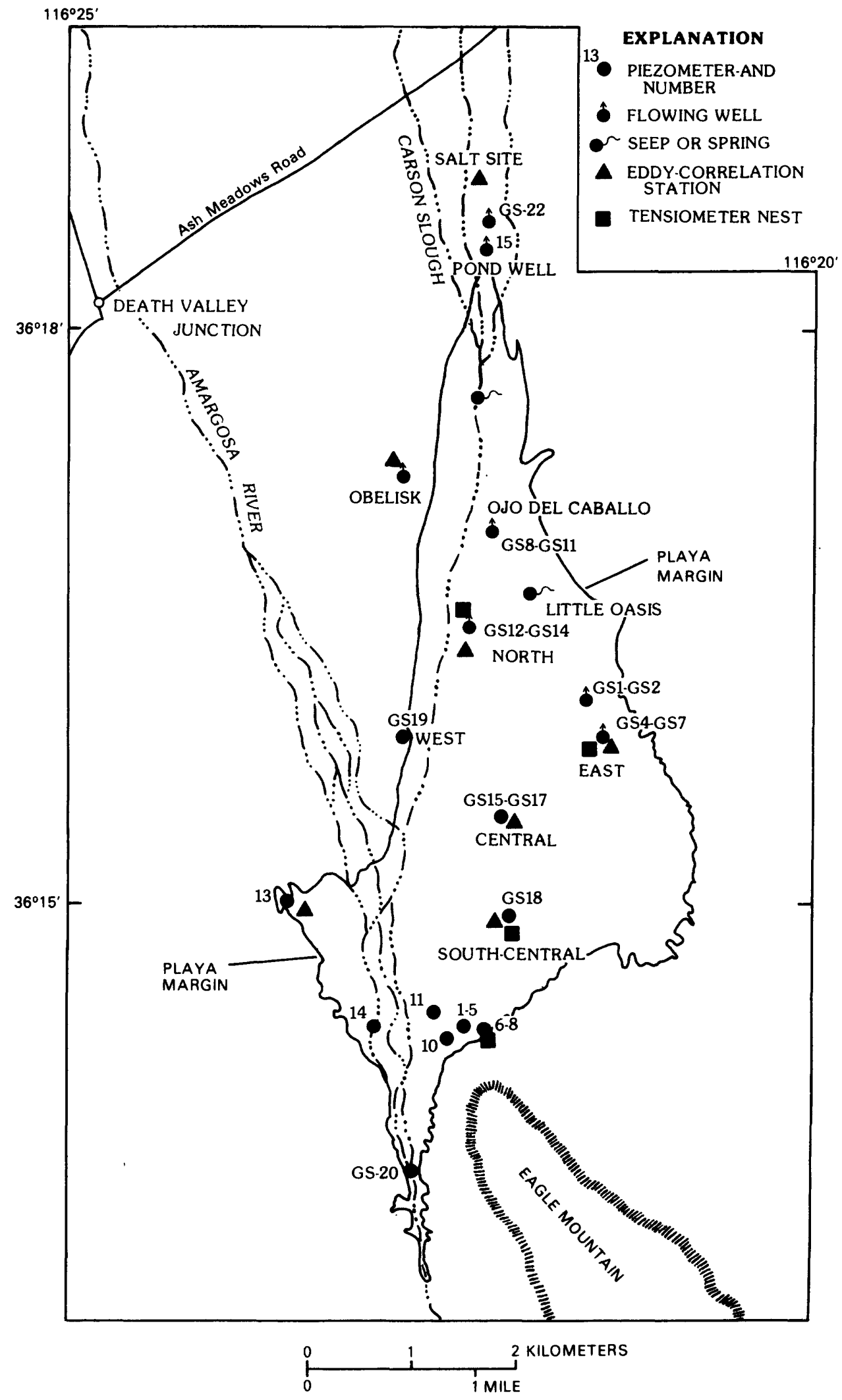

Figure 2.--Study sites at Franklin Lake playa. 


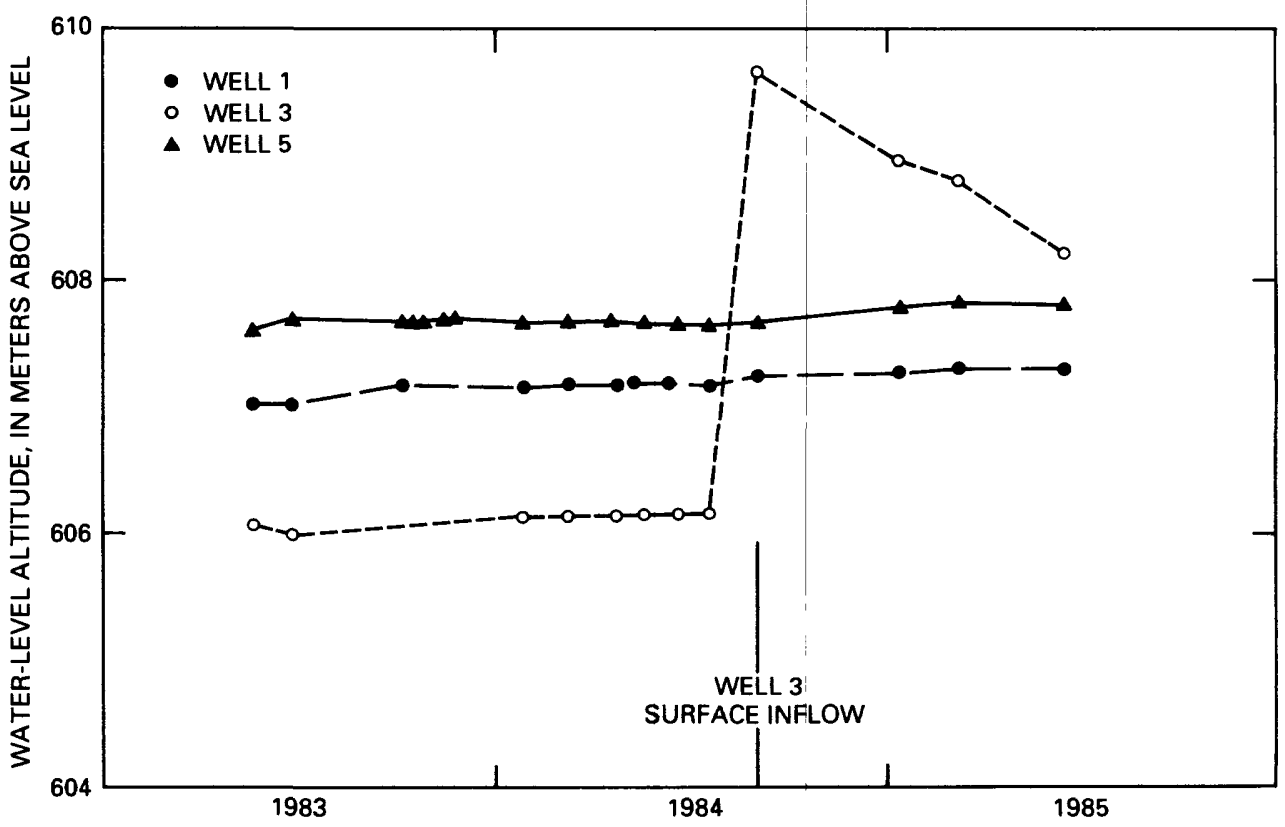

Figure 3A.--Water-level altitude in wells 1,3 , and 5 .

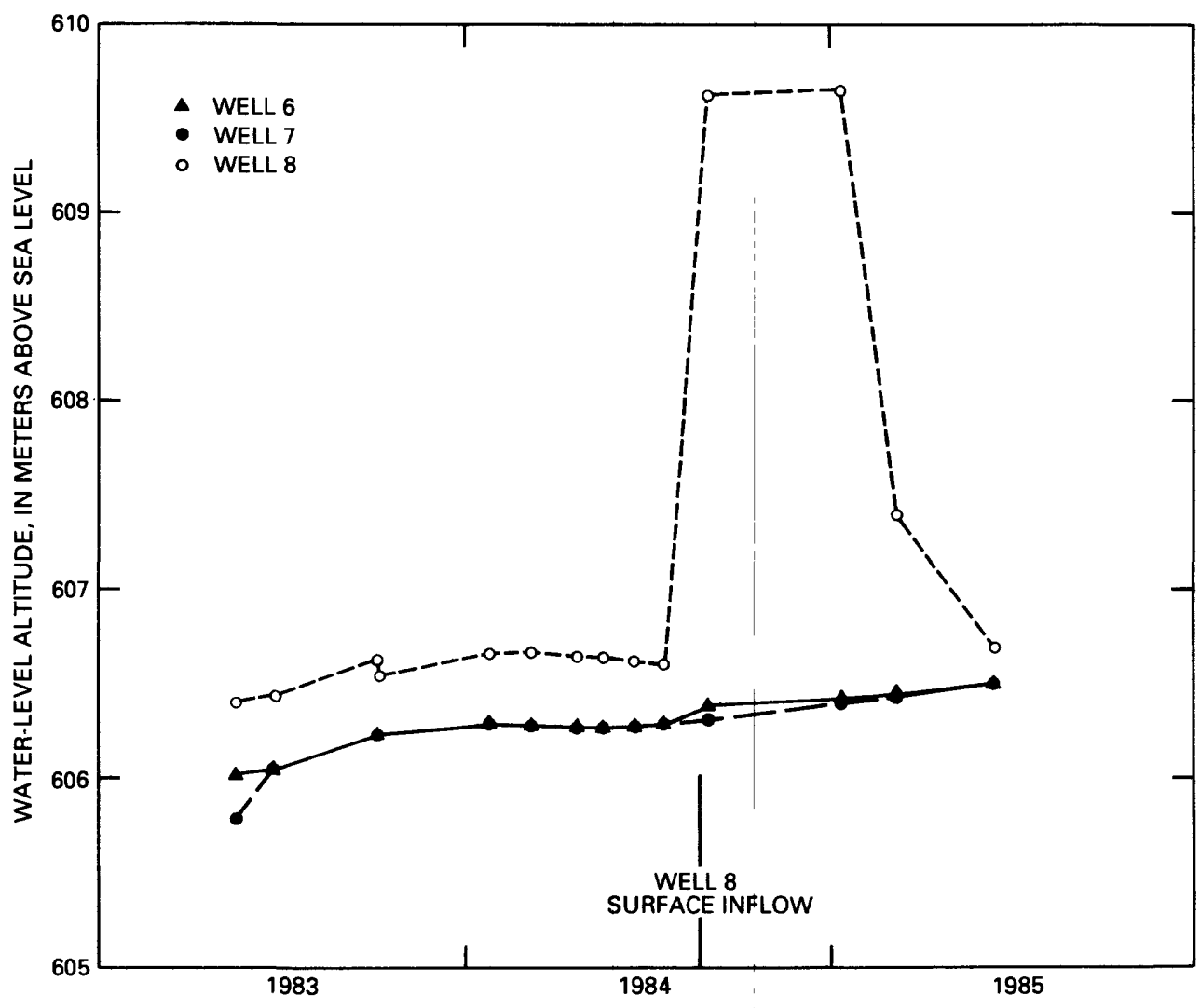

Figure $3 B .-$ Water-level altitude in wells 6,7 , and 8 . 


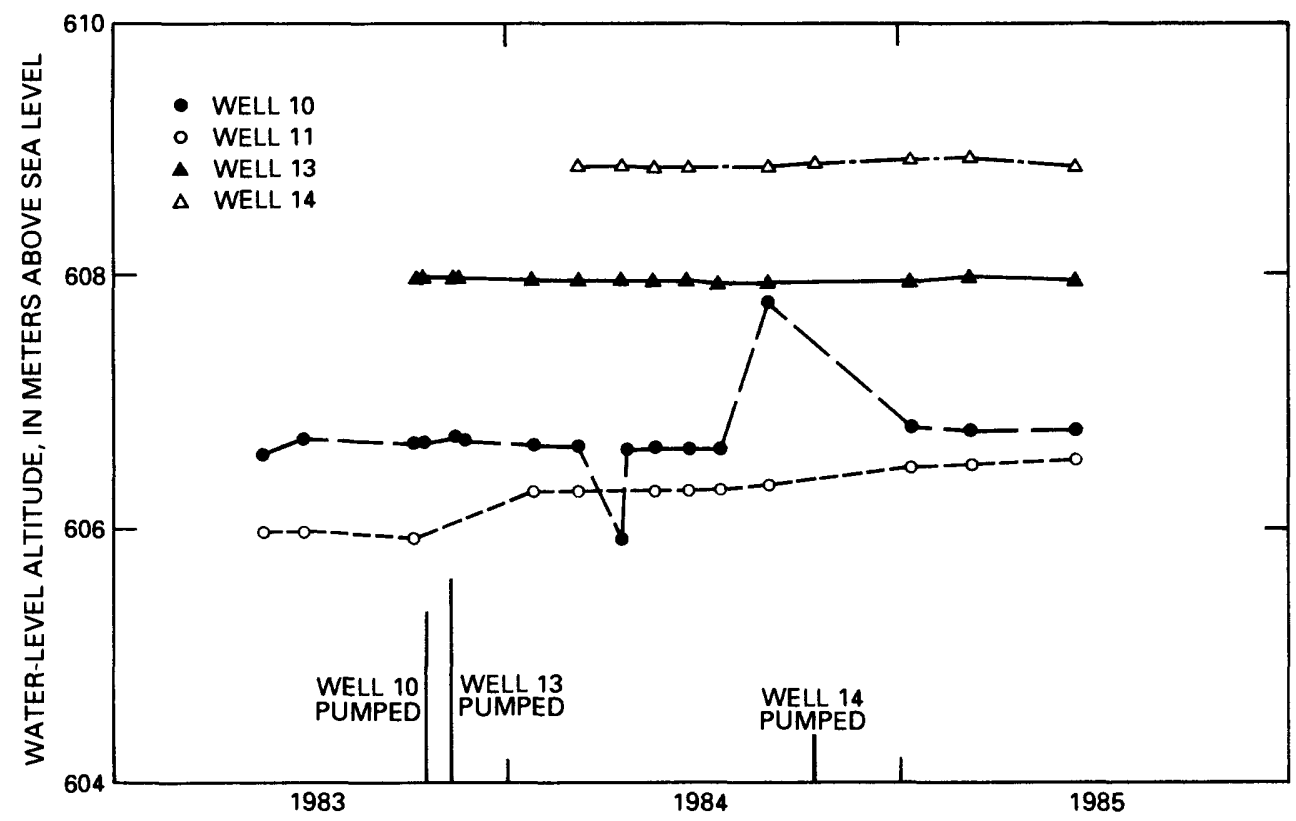

Figure 3C.--Water-level altitude in wells 10, 11, 13, and 14 .

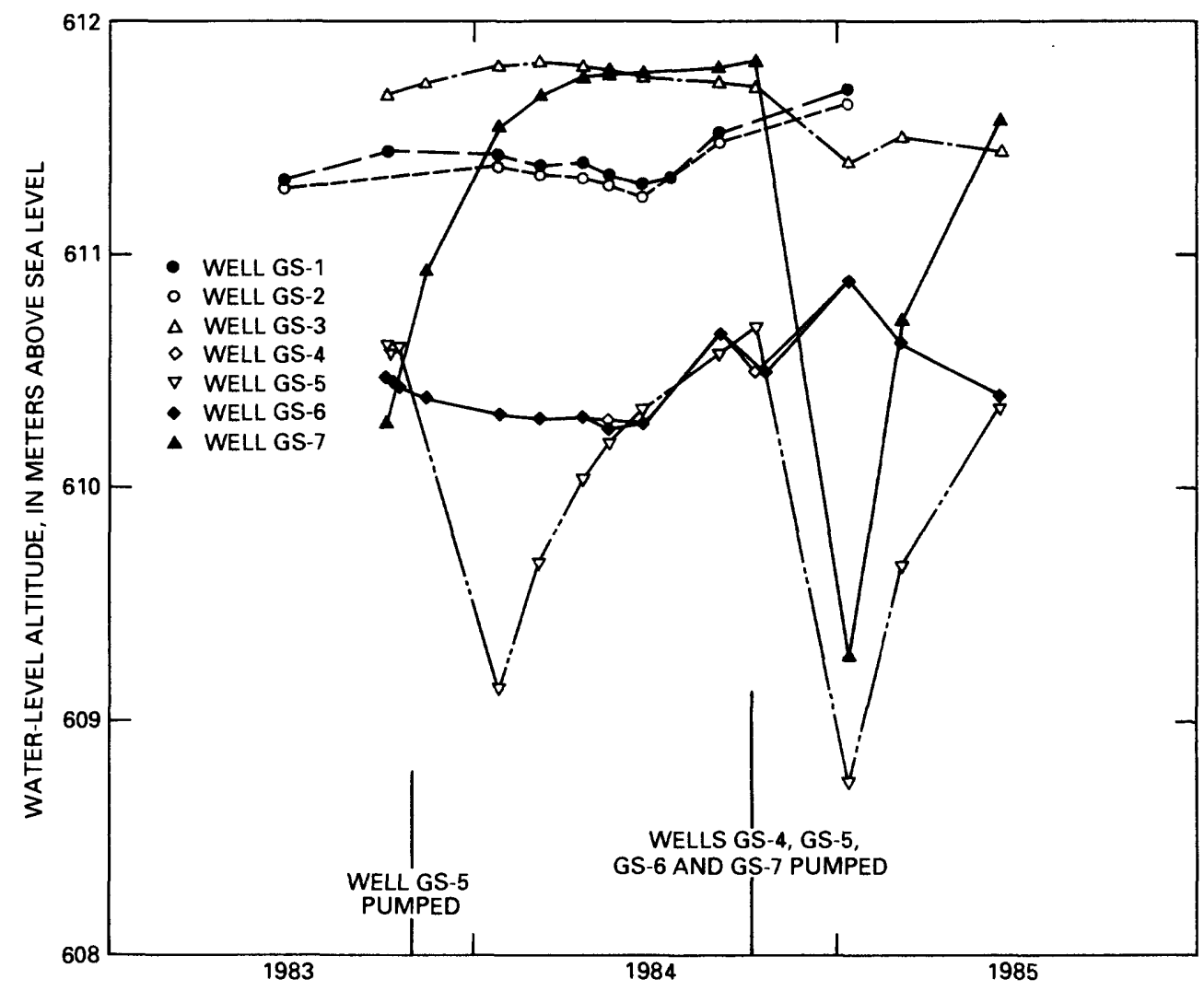

Figure 3D.--Water-level altitude in wells GS-1, GS-2, GS-3, GS-4, GS-5, GS-6, and GS-7. 


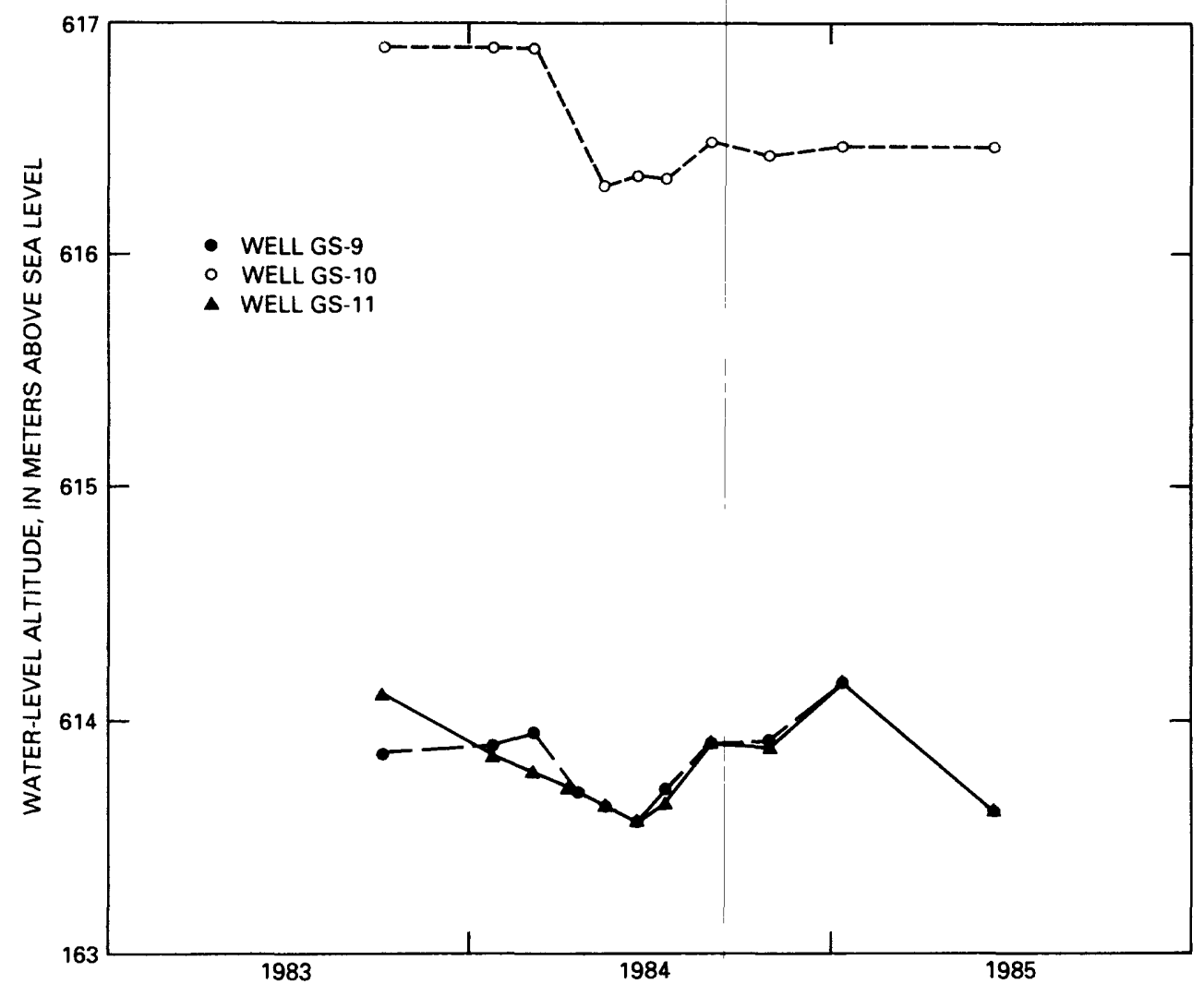

Figure 3E.--Water-level altitude in wells GS-9, GS-10, and GS-11.

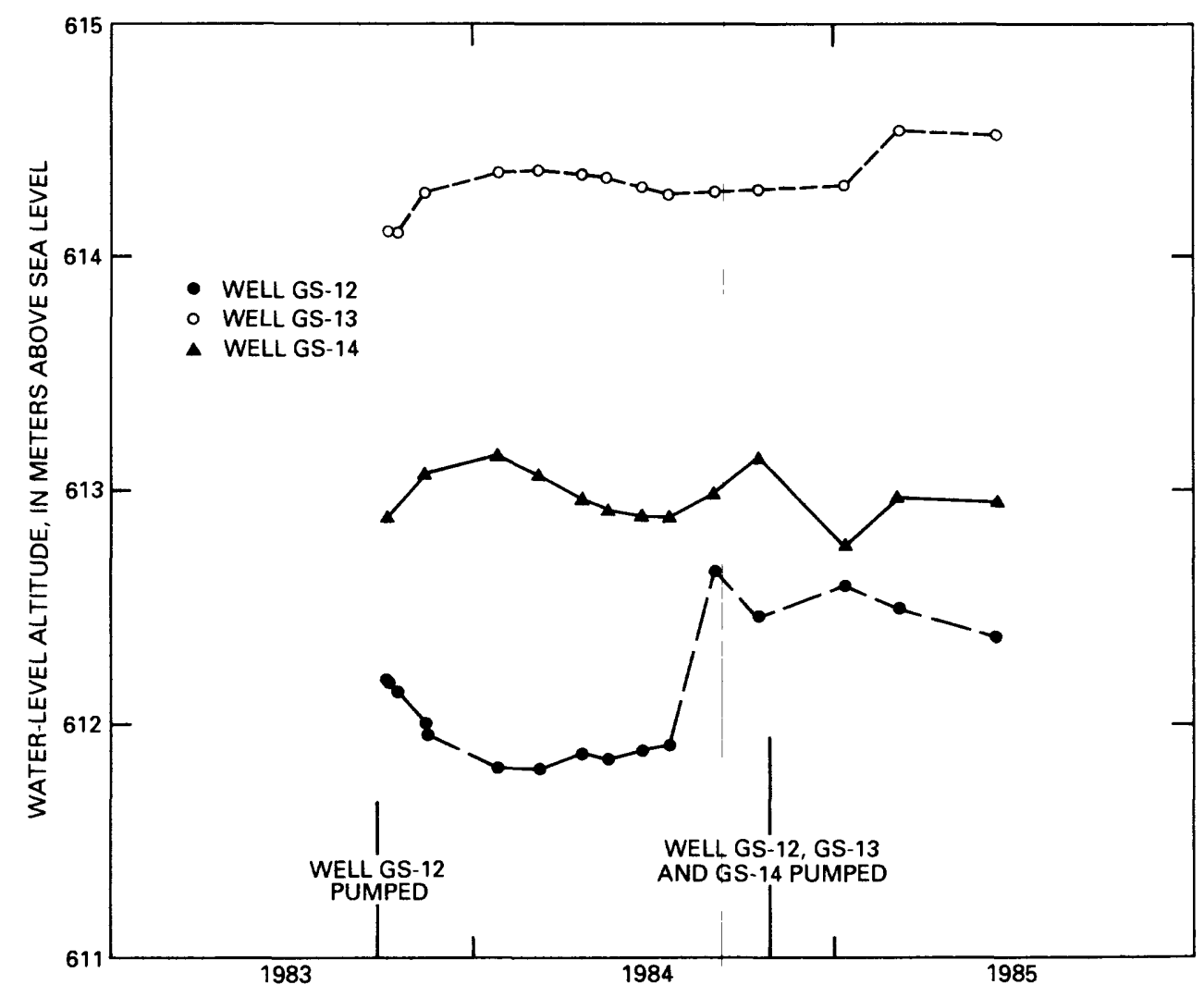

Figure 3F.--Water-level altitude in wells GS-12, GS-13, and GS-14. 


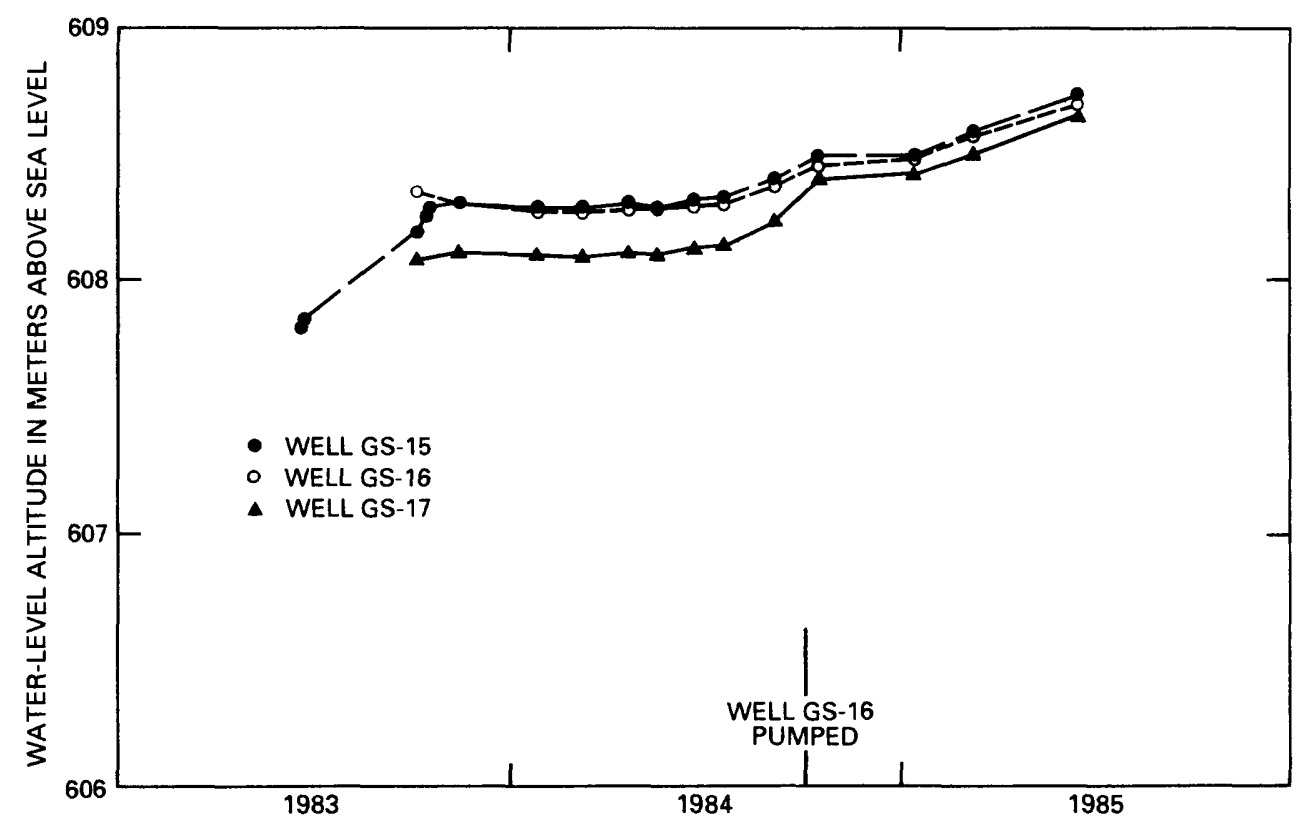

Figure 3G.--Water-level altitude in wells GS-15, GS-16, and GS-17.

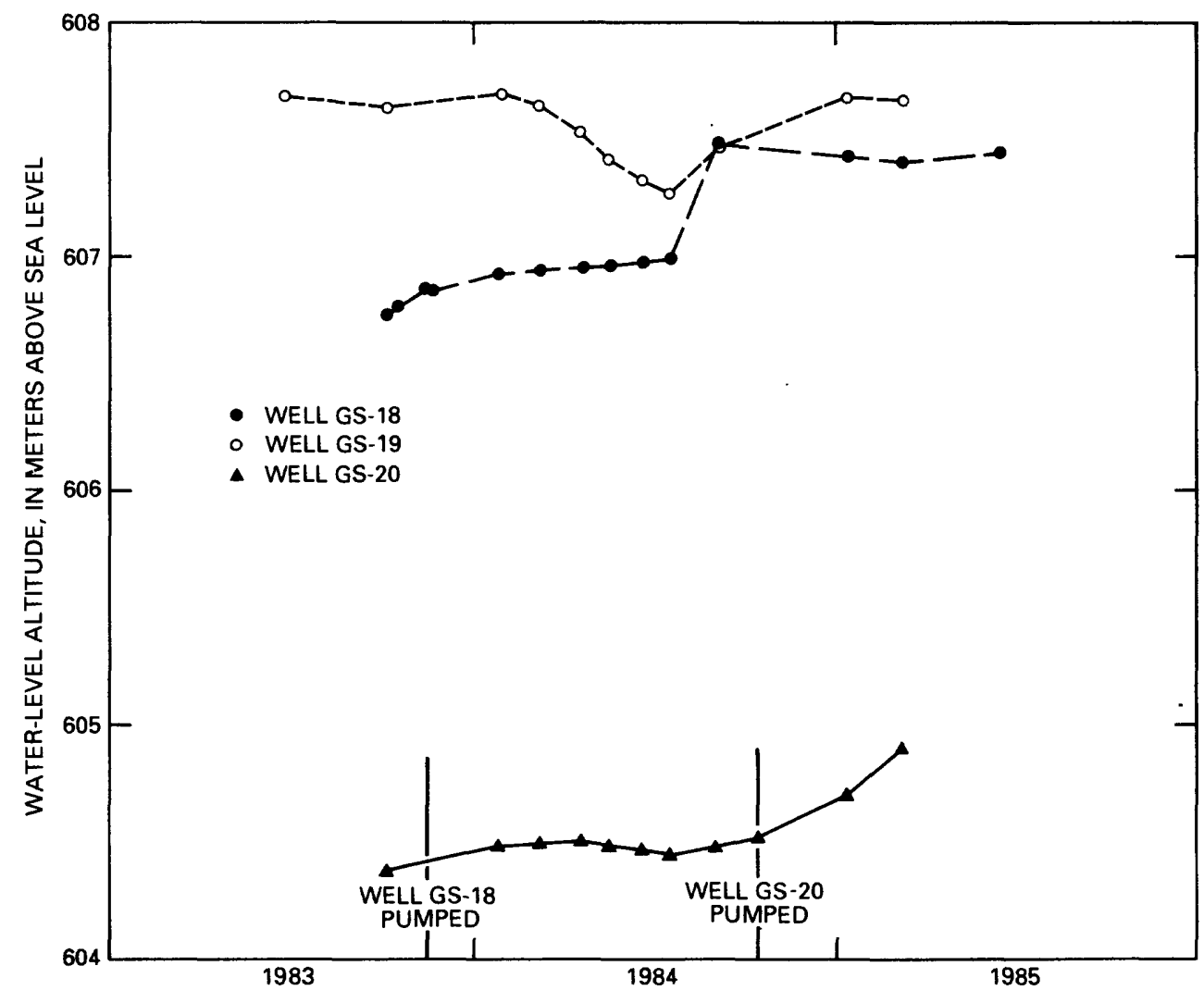

Figure 3H.--Water-level altitude in wells GS-18, GS-19, and GS-20. 


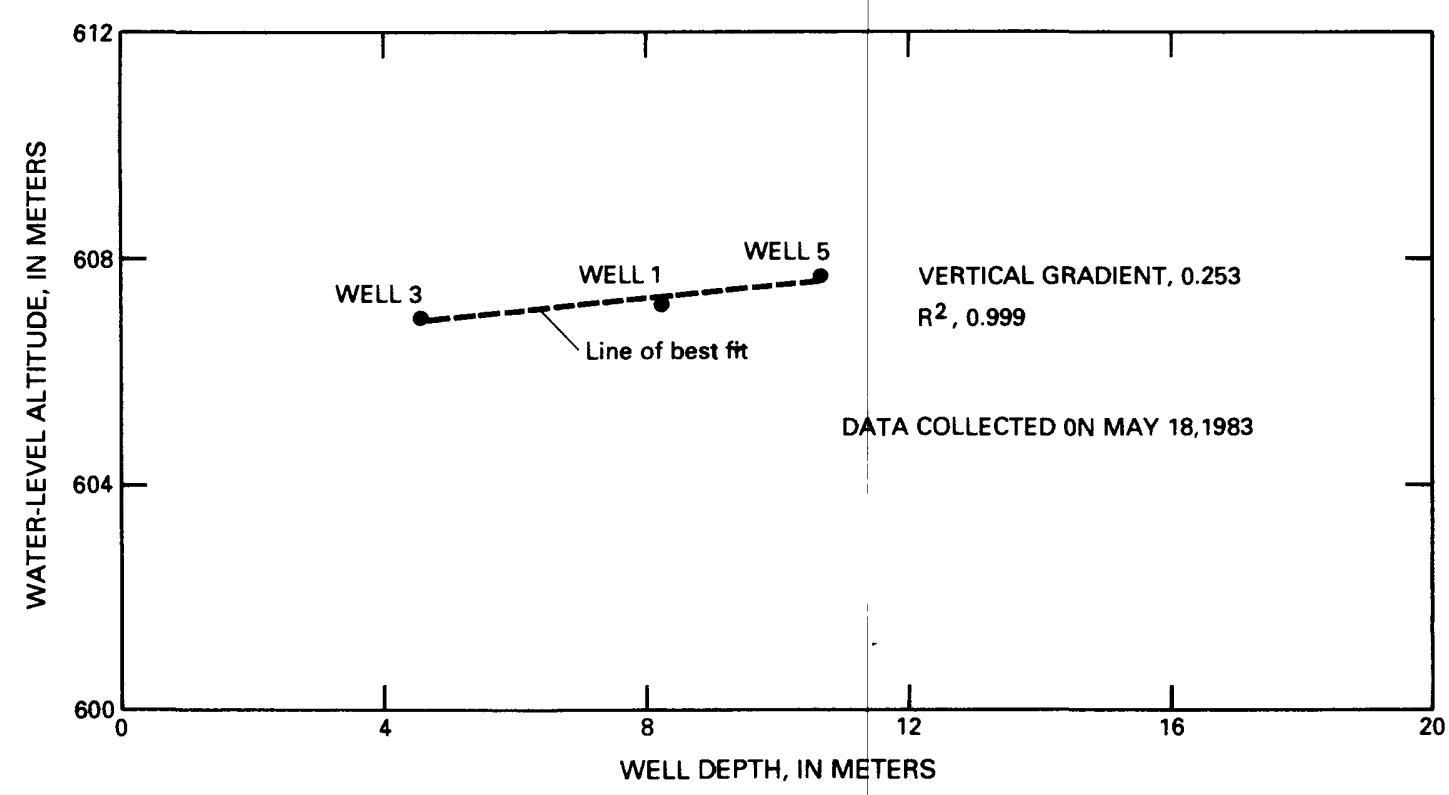

Figure 4.--Example of line of best fit through water-level-altitude data versus well-depth data obtained on May 18, 1983, for wells 1,3 , and 5 .

\section{METEOROLOGICAL DATA}

Meteorological data used to estimate potential evapotranspiration based on empirical relations were supplied by the U.S. National Weather Service, Nuclear Support Office (Douglas Soule, written commun., 1985) for weather stations located at Mercury, Nev.; Boulder City, Nev.; and Silverpeak, Nev. Mercury, Nev., is located about $60 \mathrm{~km}$ northwest of Franklin Lake playa; Boulder City, Nev., is about $140 \mathrm{~km}$ southeast; and Silverpeak, Nev., is about $200 \mathrm{~km}$ northwest. Meteorologic data for these three stations are listed in tables 1-3. The most complete record exists for Mercury, Nev.; however, pan-evaporation data do not exist for this station. 


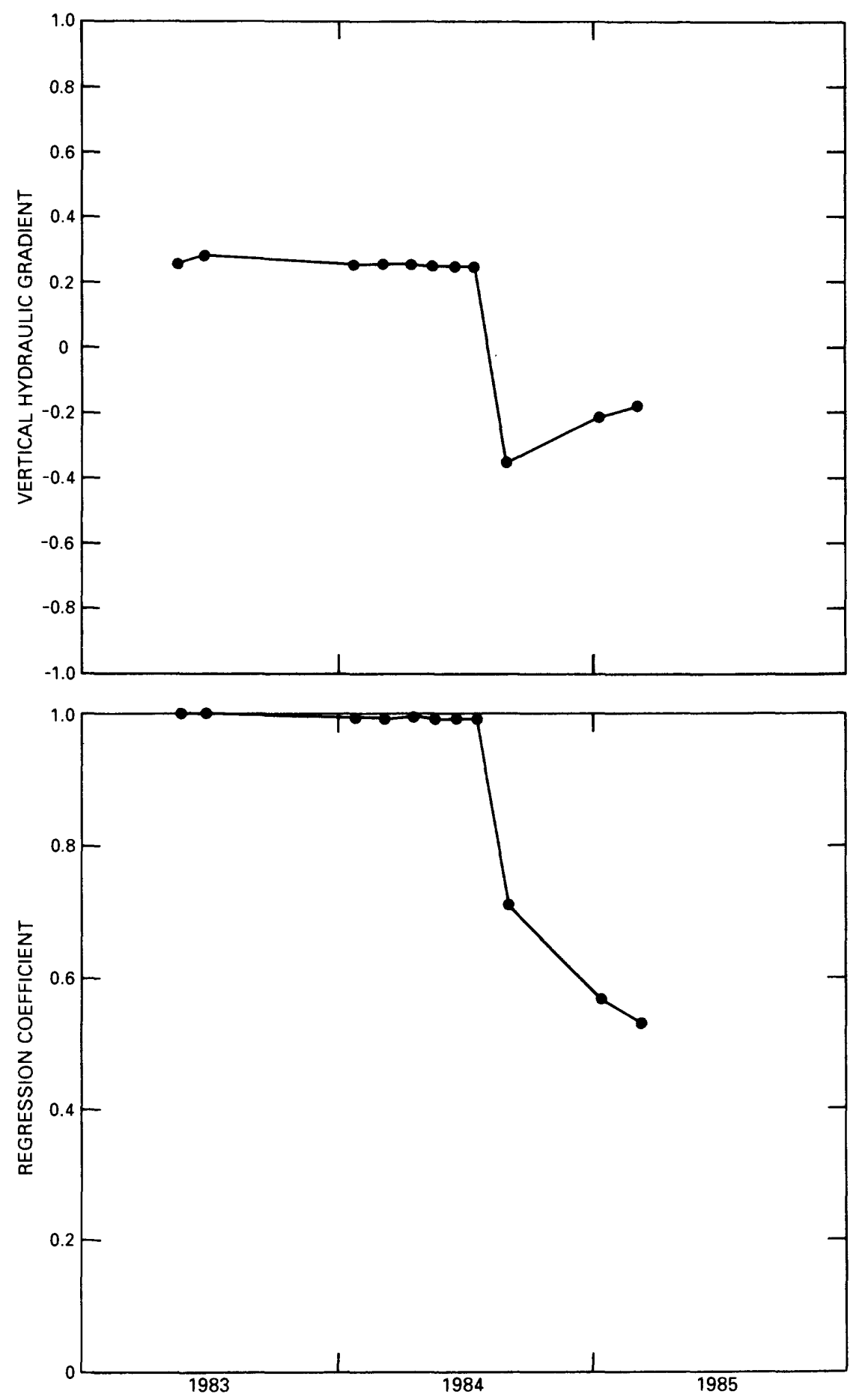

Figure 5A.--Vertical hydraulic gradient and regression coefficient for wells 1,3 , and 5 . 

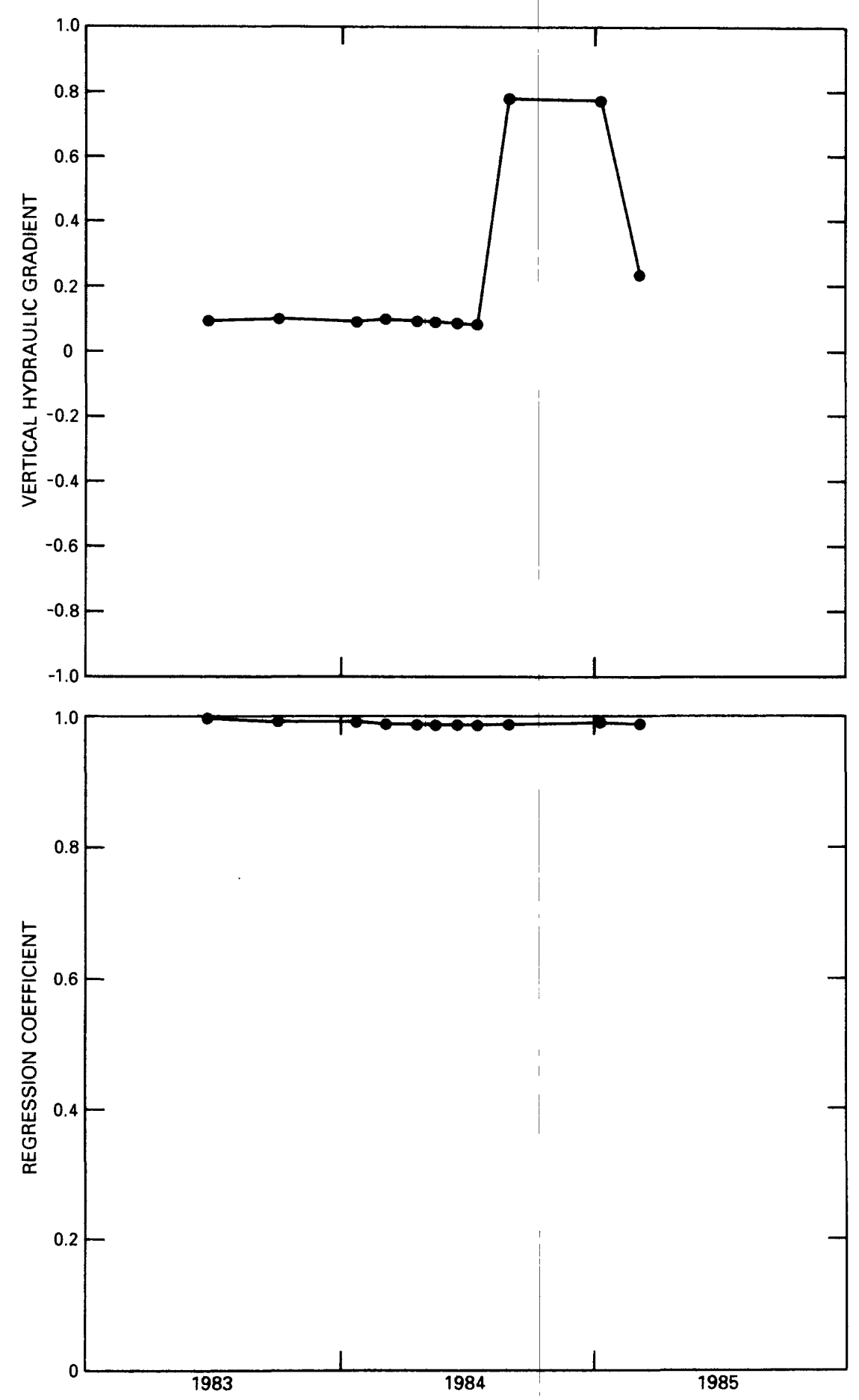

Figure 5B.--Vertical hydraulic gradient and regression coefficient for wells 6,7 , and 8 . 

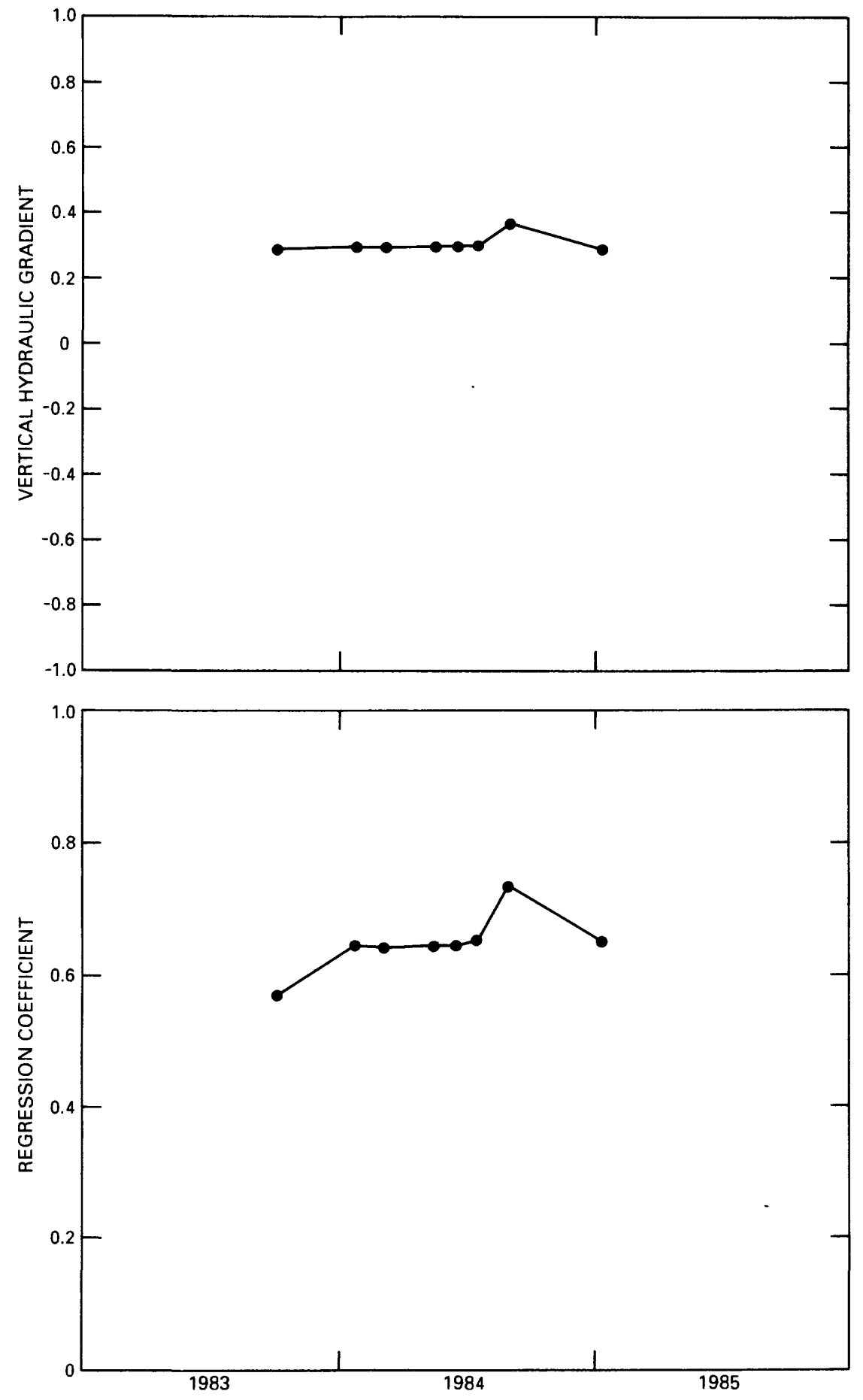

Figure 5C.--Vertical hydraulic gradient and regression coefficient for wells $4,10,11$, and GS-20. 

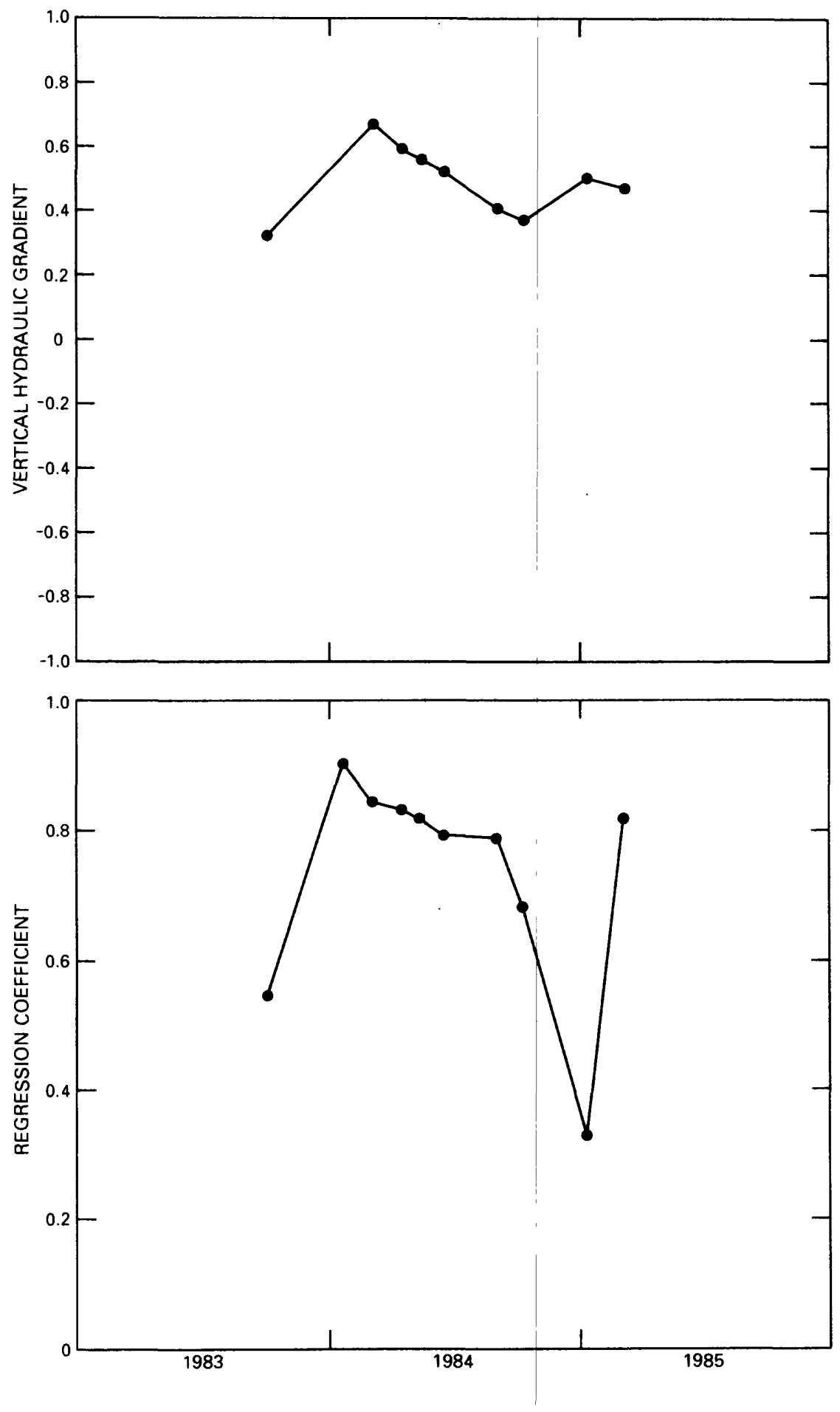

Figure 5D.--Vertical hydraulic gradient and regression coefficient for wells GS-3, GS-4, GS-5, and GS-7. 

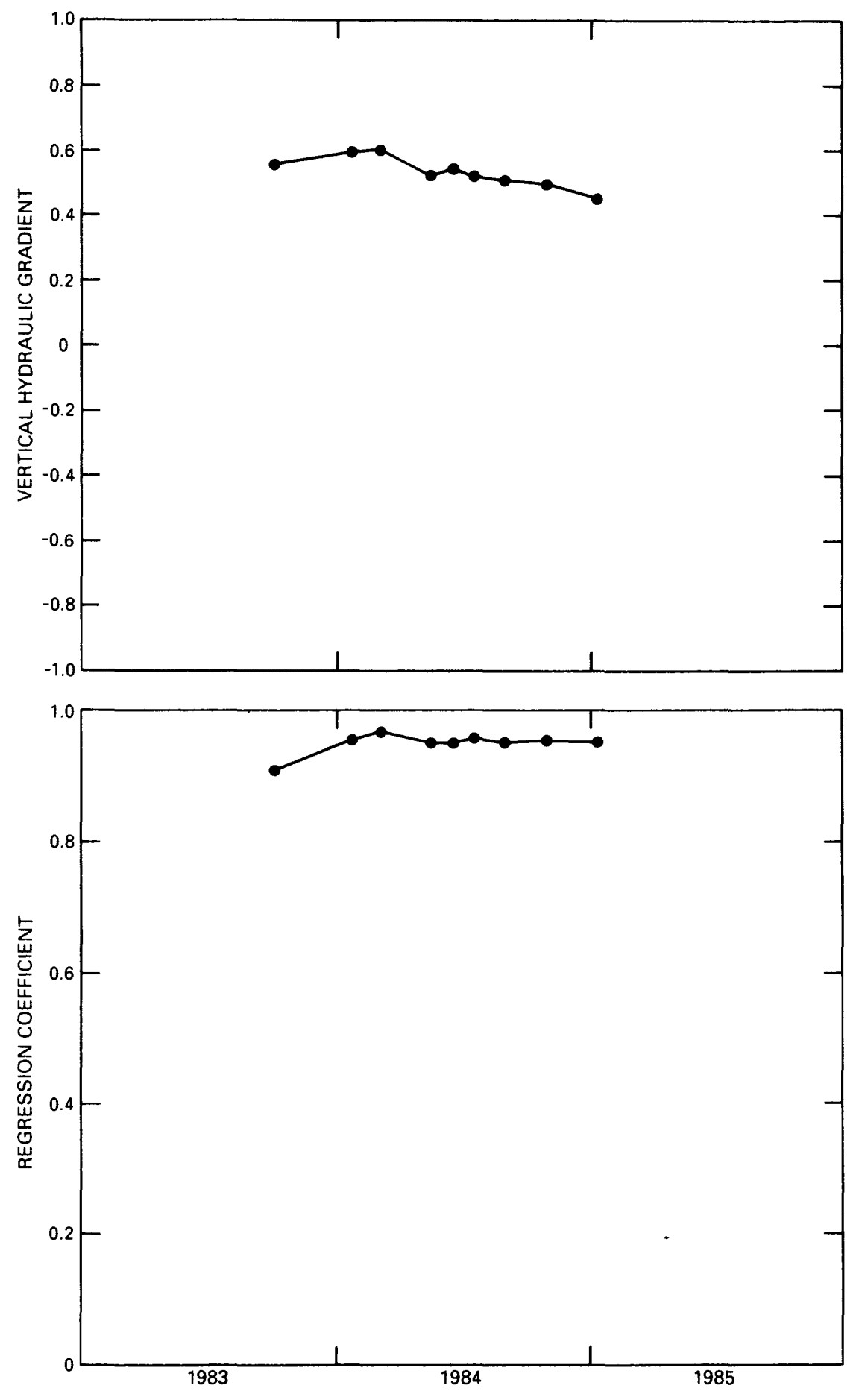

Figure 5E.--Vertical hydraulic gradient and regression coefficient for wells GS-9, GS-10, and GS-11. 

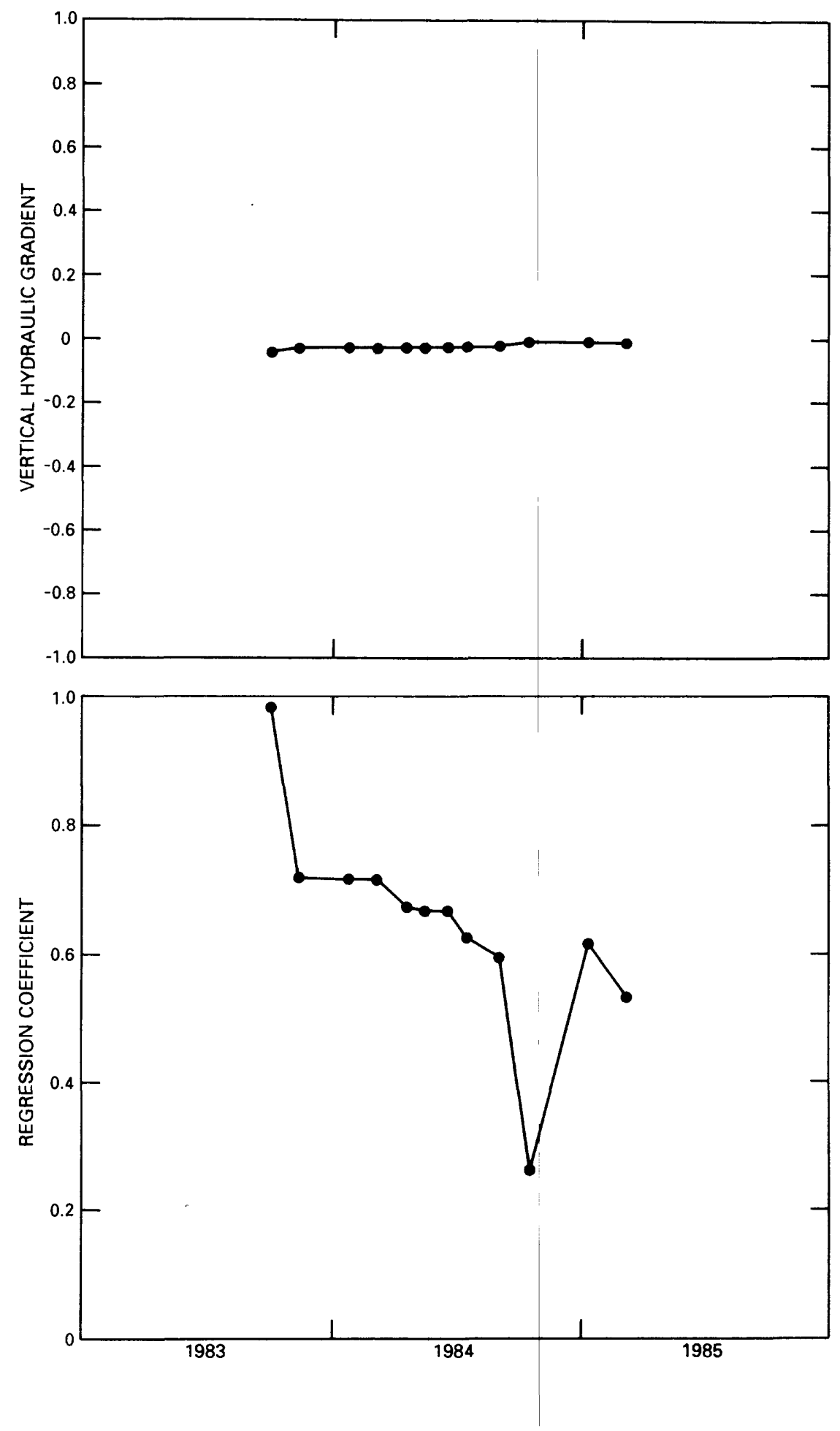

Figure 5F.--Vertical hydraulic gradient and regression coefficient for wells GS-15, GS-16, and GS-17. 

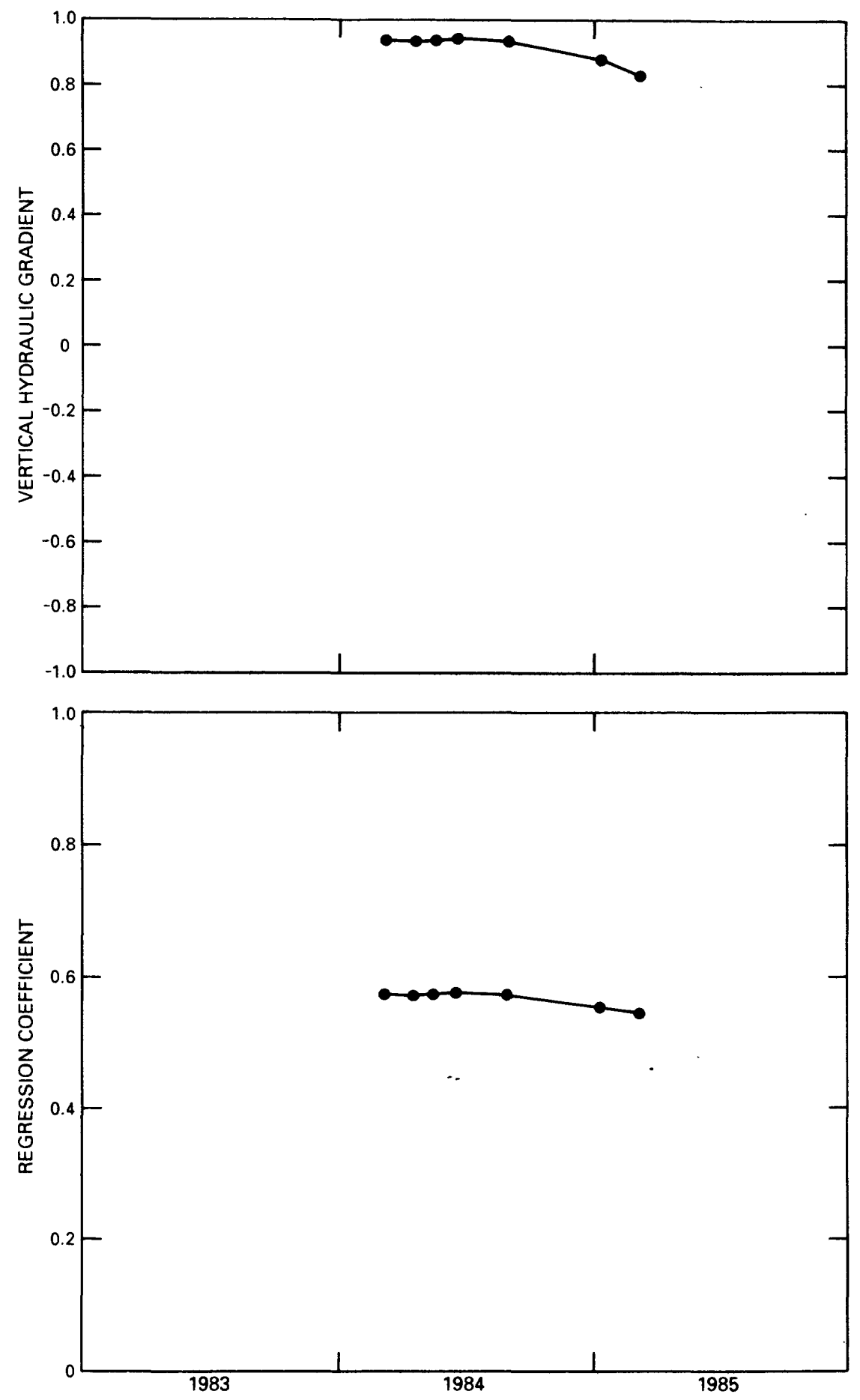

Figure 5G.--Vertical hydraulic gradient and regression coefficient for wells 13,14 , and GS-20. 


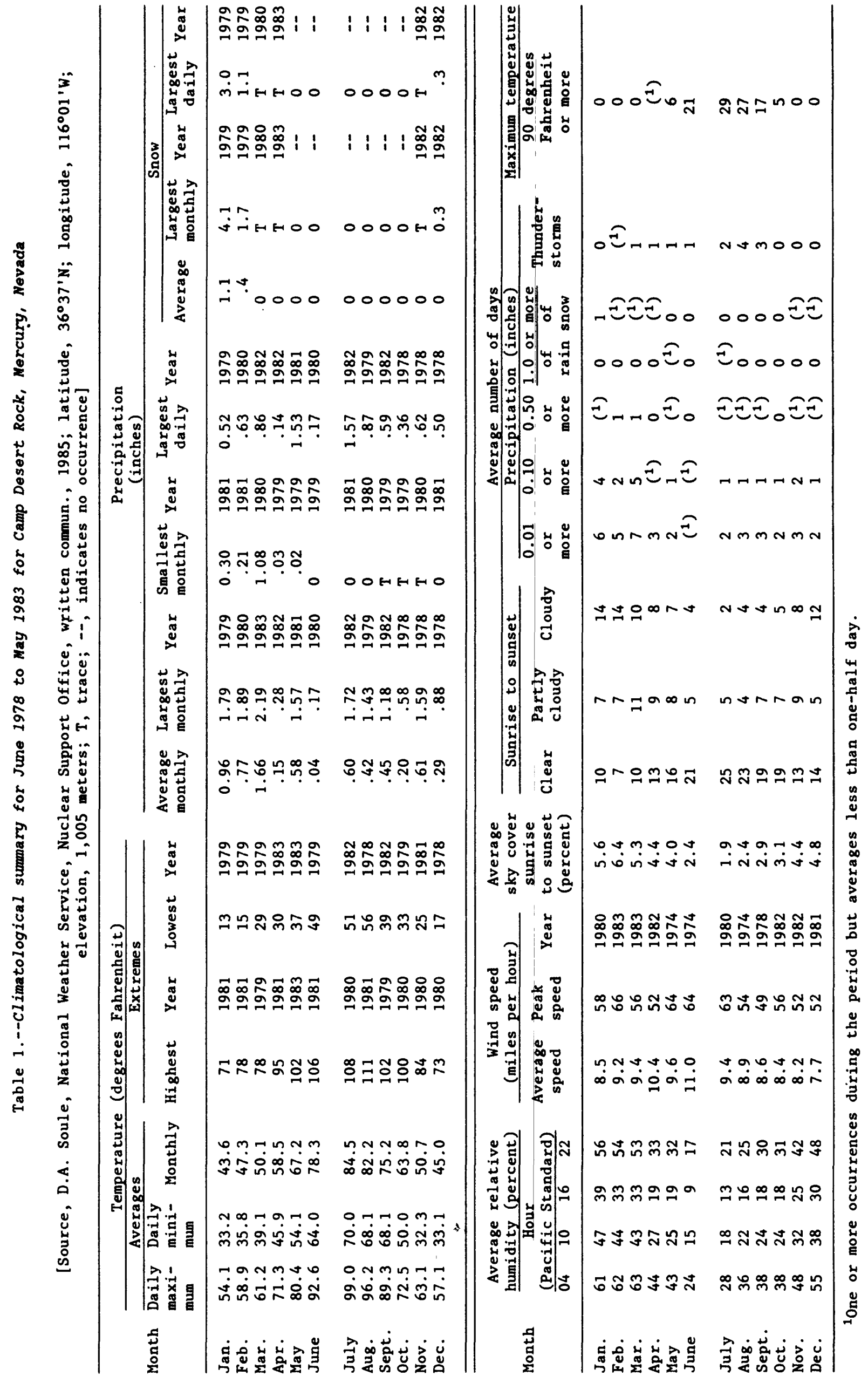


Table 2.--Summary of temperatures, wind speeds, and pan-evaporation rates for Boulder City, Nevada, 1982-83

[Source, D.A. Soule, National Weather Service, Nuclear Support Office, written commun., 1985; latitude, $35^{\circ} 55^{\prime} \mathrm{N}$; longitude, $114^{\circ} 50^{\prime} \mathrm{W}$; elevation, 770 meters; ${ }^{\circ} \mathrm{F}$, degrees Fahrenheit; mi/mo, miles per month; in/mo, inches per month; --, no measurement available]

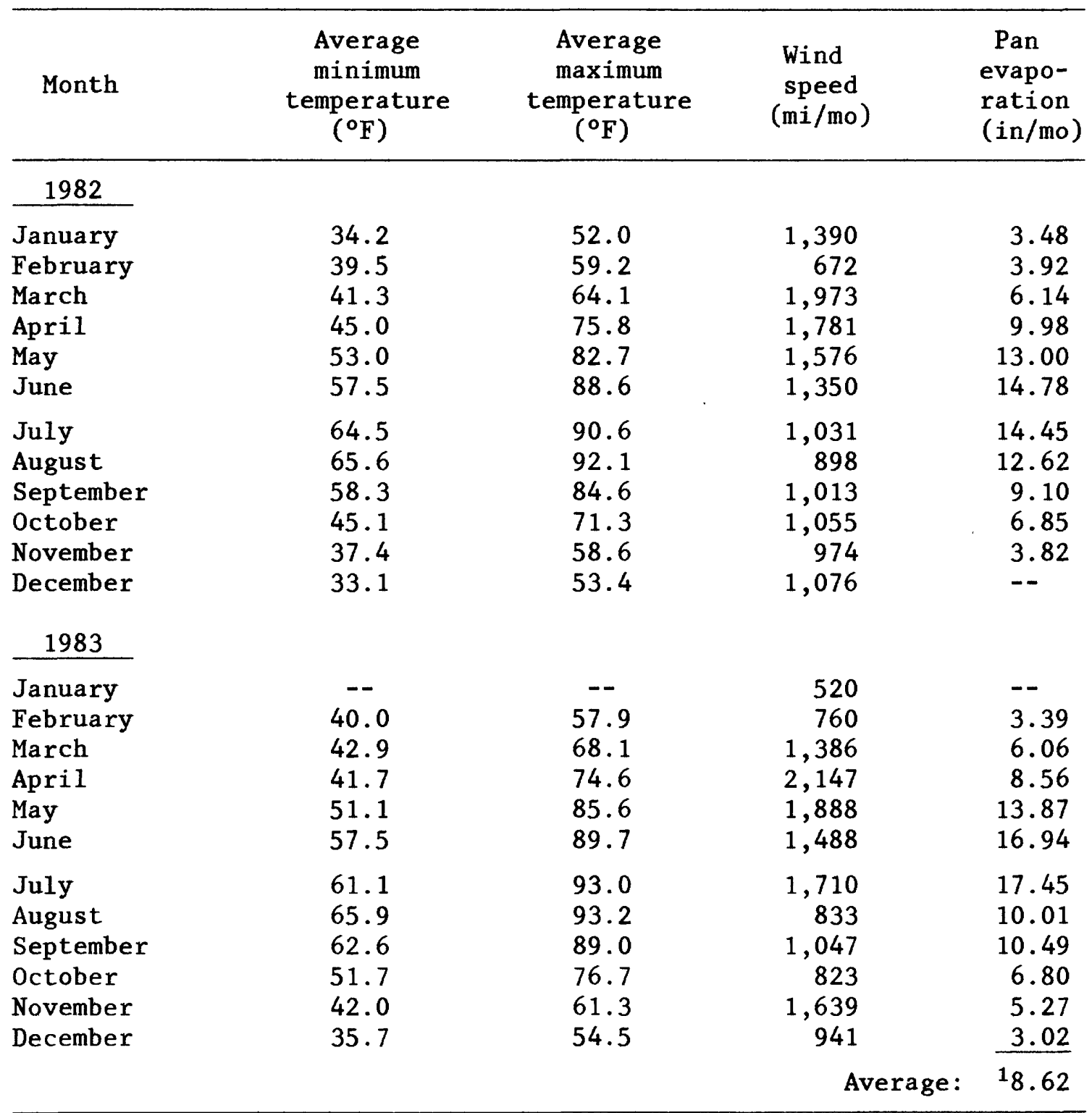

${ }^{1}$ Missing values estimated to be 3.5 inches per month. 
Table 3.--Summary of temperatures, wind speeds, and pan-evaporation rates for Silverpeak, Nevada, 1982-83

[Source, D.A. Soule, National Weather Service, Nuclear Support Office,

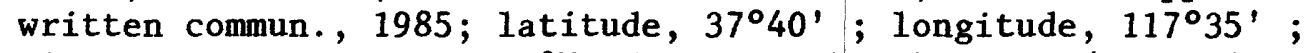
elevation, 1,300 meters; ${ }^{\circ} \mathrm{F}$, degrees Fahrenheit; mi/mo, miles per month; in/mo, inches per month; --, no measurement available]

\begin{tabular}{|c|c|c|c|c|}
\hline Month & $\begin{array}{c}\text { Average } \\
\text { minimum } \\
\text { temperature } \\
\left({ }^{\circ} \mathrm{F}\right)\end{array}$ & $\begin{array}{c}\text { Average } \\
\text { maximum } \\
\text { temperature } \\
\left({ }^{\circ} \mathrm{F}\right)\end{array}$ & $\begin{array}{l}\text { Wind } \\
\text { speed } \\
(\mathrm{mi} / \mathrm{mo})\end{array}$ & $\begin{array}{l}\text { Pan } \\
\text { evapo- } \\
\text { ration } \\
\text { (in/mo) }\end{array}$ \\
\hline \multicolumn{5}{|l|}{1982} \\
\hline $\begin{array}{l}\text { January } \\
\text { February } \\
\text { March } \\
\text { April } \\
\text { May } \\
\text { June }\end{array}$ & $\begin{array}{l}-- \\
-- \\
-- \\
-- \\
--\end{array}$ & $\begin{array}{l}-- \\
-- \\
-- \\
-- \\
-- \\
--\end{array}$ & $\begin{array}{c}-- \\
-- \\
4,095 \\
-- \\
-- \\
4,032\end{array}$ & $\begin{array}{l}-- \\
\overline{-} \\
6.90 \\
-- \\
-- \\
16.22\end{array}$ \\
\hline $\begin{array}{l}\text { July } \\
\text { August } \\
\text { September } \\
\text { October } \\
\text { November } \\
\text { December } \\
\quad 1983 \\
\end{array}$ & $\begin{array}{c}-- \\
-- \\
48.6 \\
37.8 \\
-- \\
--\end{array}$ & $\begin{array}{c}-- \\
-- \\
61.3 \\
56.7 \\
-- \\
--\end{array}$ & $\begin{array}{c}3,619 \\
3,703 \\
4,330 \\
3,278 \\
-- \\
--\end{array}$ & $\begin{array}{c}17.83 \\
16.80 \\
11.88 \\
6.36 \\
-- \\
--\end{array}$ \\
\hline $\begin{array}{l}\text { January } \\
\text { February } \\
\text { March } \\
\text { April } \\
\text { May } \\
\text { June }\end{array}$ & $\begin{array}{c}-- \\
-- \\
36.2 \\
36.8 \\
44.0 \\
50.8\end{array}$ & $\begin{array}{c}-- \\
-- \\
61.1 \\
64.8 \\
75.7 \\
84.7\end{array}$ & $\begin{array}{c}-- \\
2,874 \\
4,324 \\
5,224 \\
4,889 \\
1,629\end{array}$ & $\begin{array}{l}-- \\
-- \\
-- \\
-- \\
12.76 \\
16.59\end{array}$ \\
\hline $\begin{array}{l}\text { July } \\
\text { August } \\
\text { September } \\
\text { October } \\
\text { November } \\
\text { December }\end{array}$ & $\begin{array}{l}49.2 \\
57.2 \\
50.5 \\
41.5 \\
33.0 \\
--\end{array}$ & $\begin{array}{l}83.6 \\
81.6 \\
80.1 \\
67.1 \\
56.0 \\
--\end{array}$ & $\begin{array}{c}5,026 \\
3,537 \\
3,612 \\
2,533 \\
3,264 \\
--\end{array}$ & $\begin{array}{l}20.24 \\
13.22 \\
12.38 \\
6.18 \\
-- \\
--\end{array}$ \\
\hline
\end{tabular}




\section{Moisture Content in the Unsaturated Zone}

Evapotranspiration rates conceivably may be estimated by measuring changes in soil-moisture content in the unsaturated zone. If profiles of the soil-moisture content with depth at various times are obtained, the direction of moisture movement can be inferred from the soil-moisture gradients, and estimates may be made of losses or gains in the moisture content.

Moisture content in the unsaturated zone beneath Franklin Lake playa was determined from logging data using a soil-moisture probe (Campbell Pacific Nuclear, Mode1 503 Hydroprobe ${ }^{1}$ ). The soil-moisture probe emits neutrons that are slowed in the presence of water. Moisture content can be obtained by measuring the number of neutrons emitted and deflected back to the probe. Soil-moisture logs for wells GS-4, GS-5, GS-6, GS-15, GS-18, and GS-20 appear in figure 6. These wells were constructed using $5.27-\mathrm{cm}-$ diameter ABS plastic pipe; this construction enabled insertion of the neutron probe and permitted pumping to obtain hydrochemical samples.

The soil-moisture probe was calibrated by first obtaining cores of the shallow unsaturated zone and immediately logging the core hole using the soil-moisture probe with and without plastic casing inserted in the core hole. Moisture content of the sealed core then was measured in the laboratory using a gravimetric procedure. In addition, soil-moisture probe calibrations were made in pipe suspended in air ( 0 percent water saturation) and in capped pipe suspended in a barrel of water ( 100 percent water saturation).

Soil-moisture profiles were used to identify changes in moisture content with time in the unsaturated zone, such as from cooler winter months to hot summer months. During these periods, changes in evapotranspiration may occur, and these changes might be indicated by differences in total moisture content of the soil-moisture content profiles. This approach was used to estimate moisture-flux rates. Differences in moisture-content values for different logs for all combinations of periods are listed in table $4 A-F$. These differences divided by time were used to estimate moisture flux, E, as:

$$
E=\frac{m_{1}-m_{2}}{t_{1}-t_{2}}, t_{1}<t_{2}
$$

where $m_{1}$ is the moisture content of the soil column in centimeters of water measured at time $t_{1}$, and $m_{2}$ is the content measured at time $t_{2}$. The relation between the moisture flux and the difference in time between measurements (profiles) is shown in figure 7. A histogram for all of the fluxes listed in tables $4 A-F$ is shown in figure 8 .

${ }^{1}$ Any use of brand, firm, or trade names is for identification purposes only and does not constitute endorsement by the U.S. Geological Survey. 


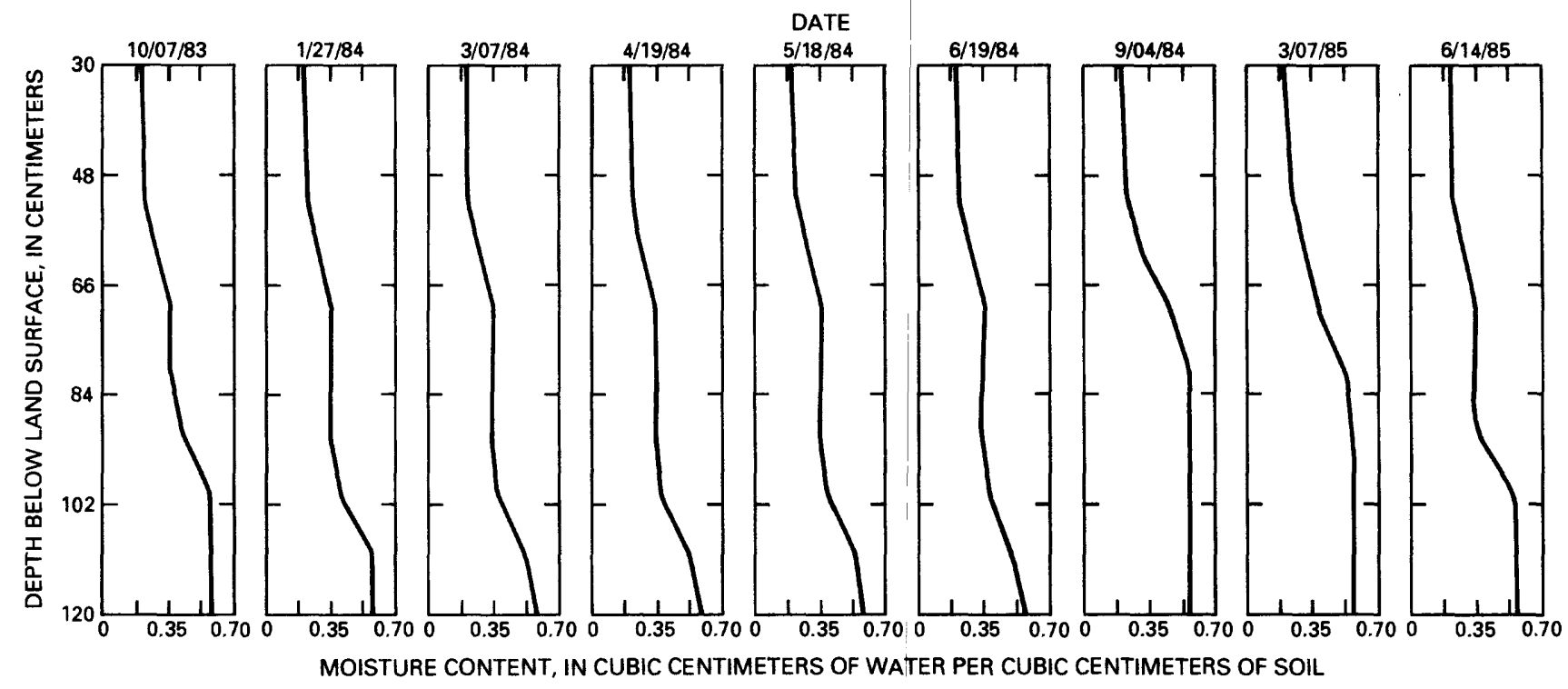

Figure 6A.--Moisture-content profiles for well GS-4.

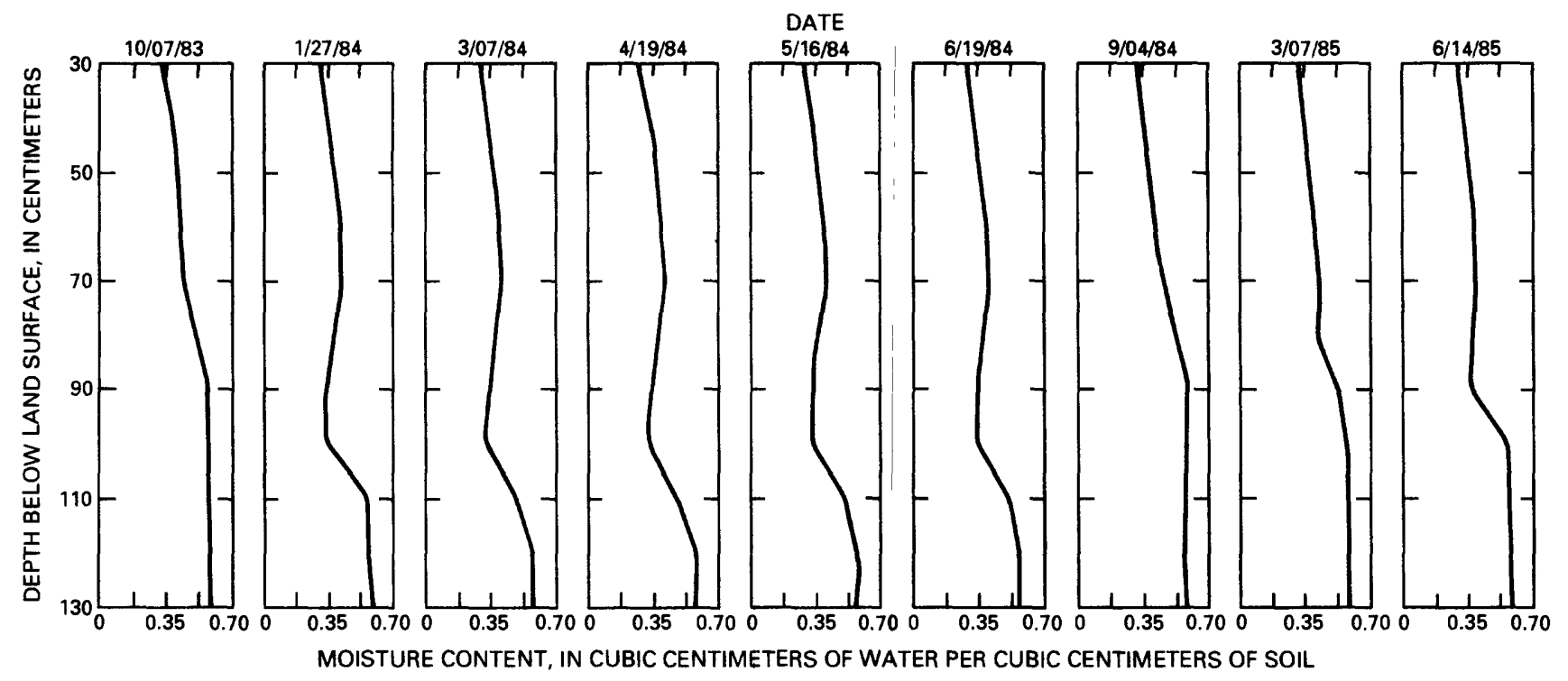

Figure 6B.--Moisture-content profiles for well GS-5. 


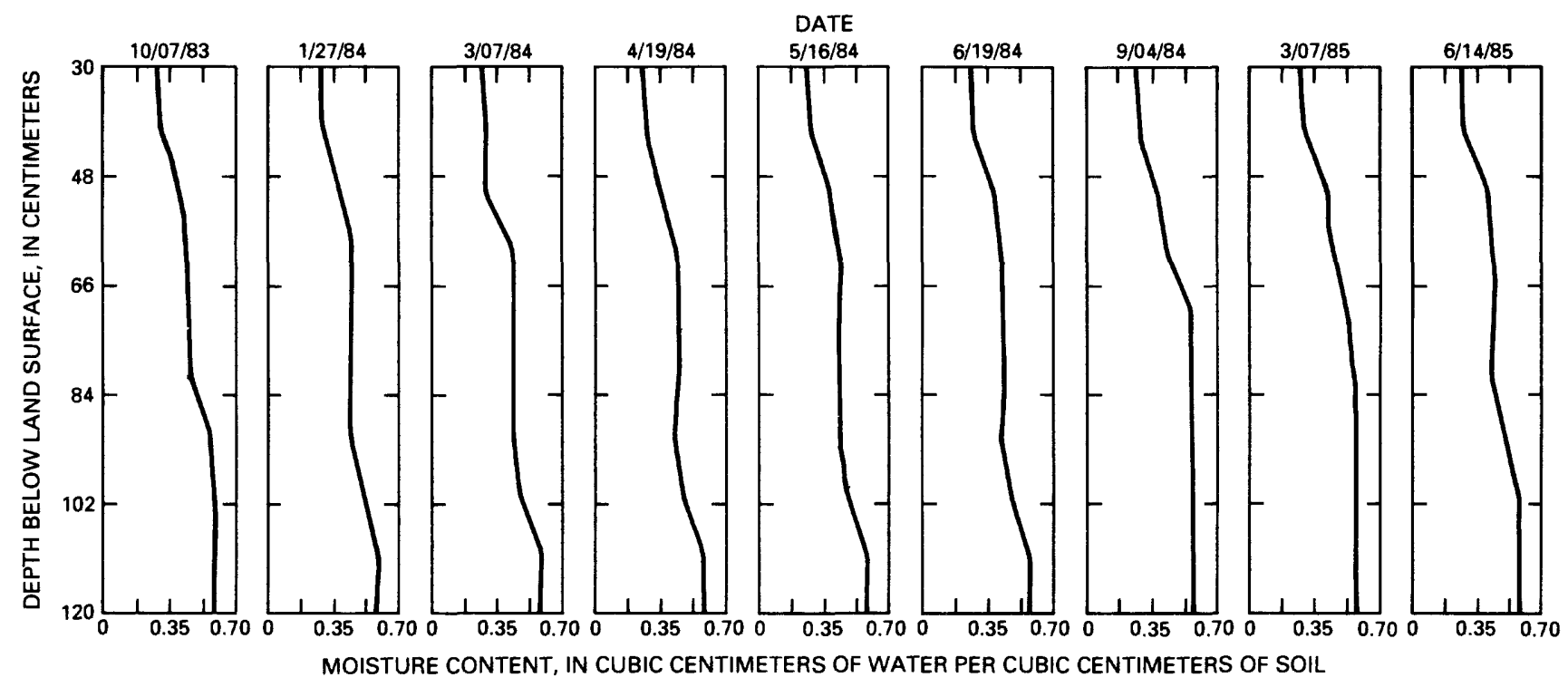

Figure 6C.--Moisture-content profiles for well GS-6.

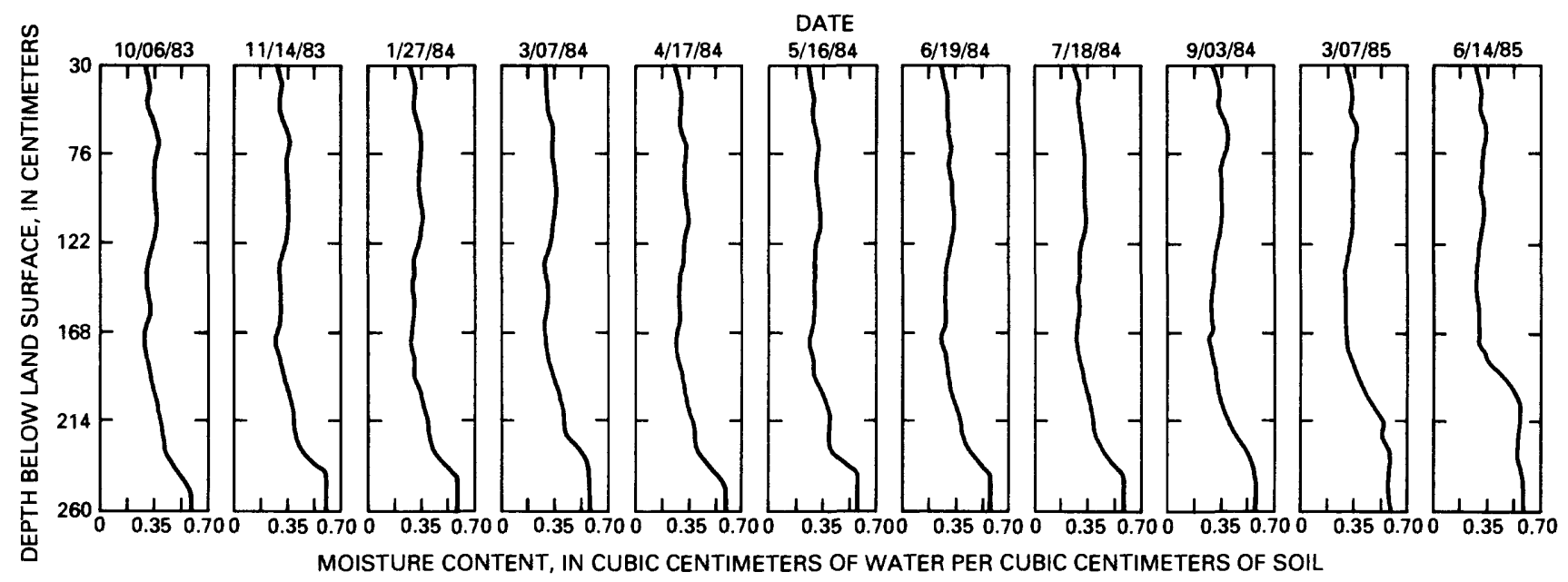

Figure 6D.--Moisture-content profiles for well GS-15. 


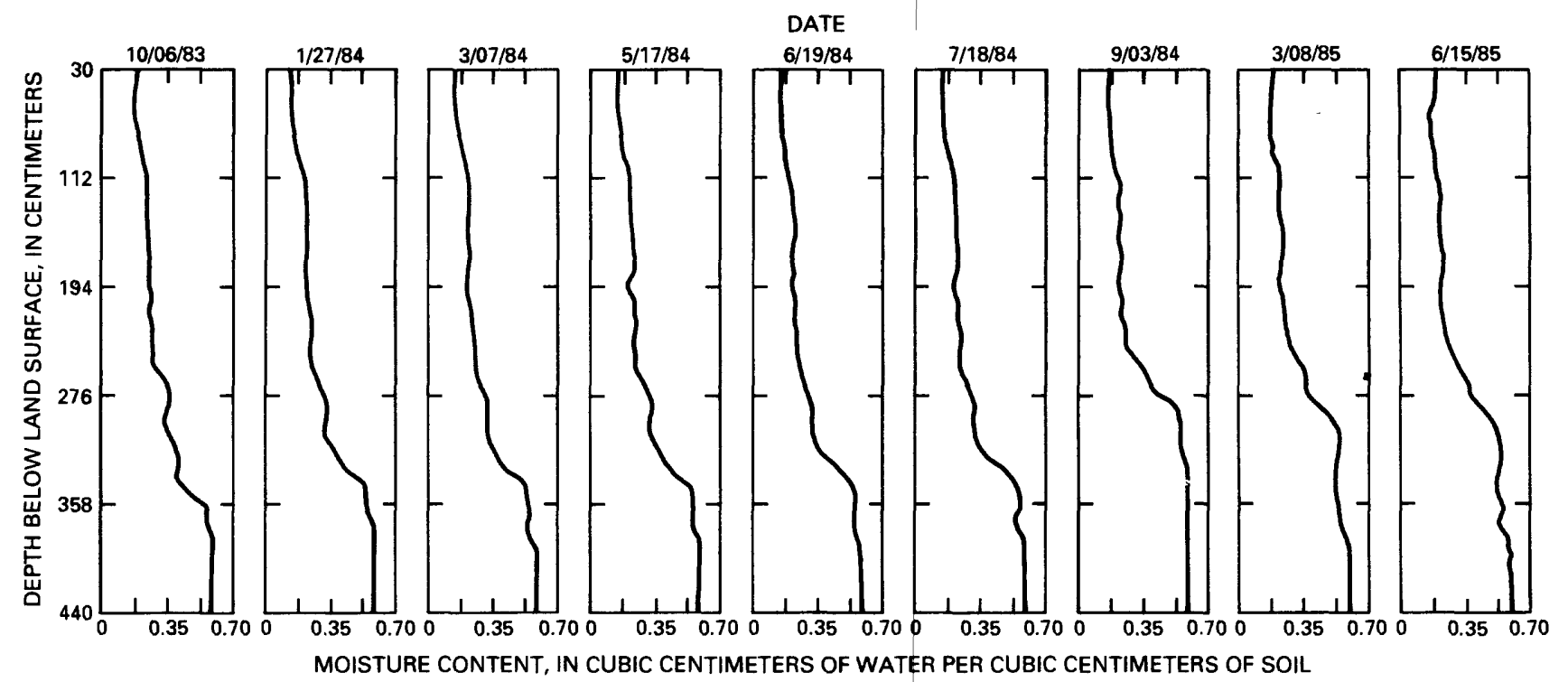

Figure 6E.--Moisture-content profiles for well GS-18.

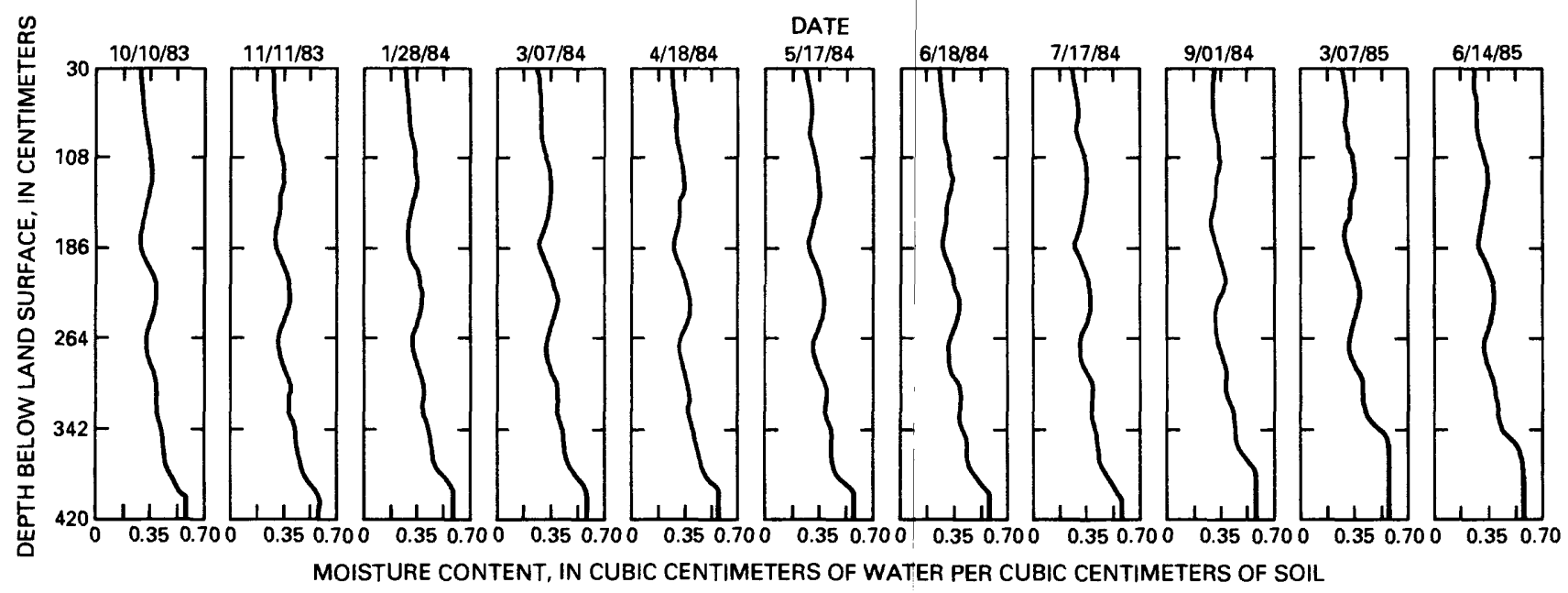

Figure 6F.--Moisture-content profiles for well GS-20. 
Table 4A.--Summary of flux values obtained from different neutron logs of well GS-4

[ $\mathrm{cm}$, centimeters, $\mathrm{cm} / \mathrm{d}$, centimeters per day]

\begin{tabular}{|c|c|c|c|c|c|c|}
\hline \multicolumn{2}{|c|}{ Period } & \multirow[b]{2}{*}{$\begin{array}{c}\text { Duration } \\
\text { (days) }\end{array}$} & \multicolumn{2}{|c|}{ Water content } & \multirow{2}{*}{$\begin{array}{c}\text { Difference } \\
\text { in water } \\
\text { content } \\
(\mathrm{cm})\end{array}$} & \multirow[b]{2}{*}{$\underset{(\mathrm{cm} / \mathrm{d})}{\text { Flux }}$} \\
\hline $\begin{array}{c}\text { First } \\
\text { date }\end{array}$ & $\begin{array}{c}\text { Second } \\
\text { date }\end{array}$ & & $\begin{array}{r}\text { First } \\
\text { date } \\
(\mathrm{cm})\end{array}$ & $\begin{array}{c}\text { Second } \\
\text { date } \\
(\mathrm{cm})\end{array}$ & & \\
\hline $\begin{array}{l}10-07-83 \\
10-07-83 \\
10-07-83 \\
10-07-83 \\
10-07-83 \\
10-07-83 \\
10-07-83 \\
10-07-83\end{array}$ & $\begin{array}{l}1-27-84 \\
3-07-84 \\
4-19-84 \\
5-18-84 \\
6-19-84 \\
9-04-84 \\
3-07-85 \\
6-14-85\end{array}$ & $\begin{array}{l}112 \\
152 \\
195 \\
224 \\
256 \\
333 \\
516 \\
615\end{array}$ & $\begin{array}{l}15.49 \\
15.49 \\
15.49 \\
15.49 \\
15.49 \\
15.49 \\
15.49 \\
15.49\end{array}$ & $\begin{array}{l}15.15 \\
14.81 \\
14.59 \\
14.92 \\
14.85 \\
19.22 \\
18.01 \\
15.95\end{array}$ & $\begin{array}{r}-0.34 \\
-.68 \\
-.90 \\
-.56 \\
-.64 \\
3.73 \\
2.52 \\
.46\end{array}$ & $\begin{array}{r}-0.003 \\
-.004 \\
-.005 \\
-.003 \\
-.003 \\
.011 \\
.005 \\
.001\end{array}$ \\
\hline $\begin{array}{l}1-27-84 \\
1-27-84 \\
1-27-84 \\
1-27-84 \\
1-27-84 \\
1-27-84 \\
1-27-84\end{array}$ & $\begin{array}{l}3-07-84 \\
4-19-84 \\
5-18-84 \\
6-19-84 \\
9-04-84 \\
3-07-85 \\
6-14-85\end{array}$ & $\begin{array}{r}40 \\
83 \\
112 \\
144 \\
220 \\
405 \\
504\end{array}$ & $\begin{array}{l}15.15 \\
15.15 \\
15.15 \\
15.15 \\
15.15 \\
15.15 \\
15.15\end{array}$ & $\begin{array}{l}14.81 \\
14.59 \\
14.92 \\
14.85 \\
19.22 \\
18.01 \\
15.95\end{array}$ & $\begin{array}{r}-.34 \\
-.56 \\
-.23 \\
-.30 \\
4.07 \\
2.85 \\
.80\end{array}$ & $\begin{array}{r}-.009 \\
-.007 \\
-.002 \\
-.002 \\
.018 \\
.007 \\
.002\end{array}$ \\
\hline $\begin{array}{l}3-07-84 \\
3-07-84 \\
3-07-84 \\
3-07-84 \\
3-07-84 \\
3-07-84\end{array}$ & $\begin{array}{l}4-19-84 \\
5-18-84 \\
6-19-84 \\
9-04-84 \\
3-07-85 \\
6-14-85\end{array}$ & $\begin{array}{r}42 \\
71 \\
103 \\
180 \\
365 \\
464\end{array}$ & $\begin{array}{l}14.81 \\
14.81 \\
14.81 \\
14.81 \\
14.81 \\
14.81\end{array}$ & $\begin{array}{l}14.59 \\
14.92 \\
14.85 \\
19.22 \\
18.01 \\
15.95\end{array}$ & $\begin{array}{r}-.22 \\
.12 \\
.04 \\
4.41 \\
3.20 \\
1.14\end{array}$ & $\begin{array}{r}-.005 \\
.002 \\
.000 \\
.024 \\
.009 \\
.002\end{array}$ \\
\hline $\begin{array}{l}4-19-84 \\
4-19-84 \\
4-19-84 \\
4-19-84 \\
4-19-84\end{array}$ & $\begin{array}{l}5-18-84 \\
6-19-84 \\
9-04-84 \\
3-07-85 \\
6-14-85\end{array}$ & $\begin{array}{r}29 \\
61 \\
137 \\
322 \\
421\end{array}$ & $\begin{array}{l}14.59 \\
14.59 \\
14.59 \\
14.59 \\
14.59\end{array}$ & $\begin{array}{l}14.92 \\
14.85 \\
19.22 \\
18.01 \\
15.95\end{array}$ & $\begin{array}{r}.34 \\
.26 \\
4.63 \\
3.42 \\
1.36\end{array}$ & $\begin{array}{l}.012 \\
.004 \\
.034 \\
.011 \\
.003\end{array}$ \\
\hline $\begin{array}{l}5-18-84 \\
5-18-84 \\
5-18-84 \\
5-18-84\end{array}$ & $\begin{array}{l}6-19-84 \\
9-04-84 \\
3-07-85 \\
6-14-85\end{array}$ & $\begin{array}{r}32 \\
108 \\
293 \\
392\end{array}$ & $\begin{array}{l}14.92 \\
14.92 \\
14.92 \\
14.92\end{array}$ & $\begin{array}{l}14.85 \\
19.22 \\
18.01 \\
15.95\end{array}$ & $\begin{array}{l}-.08 \\
4.30 \\
3.08 \\
1.03\end{array}$ & $\begin{array}{r}-.002 \\
.039 \\
.011 \\
.003\end{array}$ \\
\hline $\begin{array}{l}6-19-84 \\
6-19-84 \\
6-19-84\end{array}$ & $\begin{array}{l}9-04-84 \\
3-07-85 \\
6-14-85\end{array}$ & $\begin{array}{r}76 \\
261 \\
360\end{array}$ & $\begin{array}{l}14.85 \\
14.85 \\
14.85\end{array}$ & $\begin{array}{l}19.22 \\
18.01 \\
15.95\end{array}$ & $\begin{array}{l}4.37 \\
3.16 \\
1.10\end{array}$ & $\begin{array}{l}.057 \\
.012 \\
.003\end{array}$ \\
\hline $\begin{array}{l}9-04-84 \\
9-04-84\end{array}$ & $\begin{array}{l}3-07-85 \\
6-14-85\end{array}$ & $\begin{array}{l}184 \\
283\end{array}$ & $\begin{array}{l}19.22 \\
19.22\end{array}$ & $\begin{array}{l}18.01 \\
15.95\end{array}$ & $\begin{array}{l}-1.22 \\
-3.27\end{array}$ & $\begin{array}{l}-.007 \\
-.012\end{array}$ \\
\hline $3-07-85$ & $6-14-85$ & 99 & 18.01 & 15.95 & -2.05 & -.021 \\
\hline
\end{tabular}


Table 4B.--Summary of flux values obtained from different neutron logs of well GS-5

[ $\mathrm{cm}$, centimeters, $\mathrm{cm} / \mathrm{d}$, centimeters per day]

\begin{tabular}{|c|c|c|c|c|c|c|}
\hline Per & & & Water & content & Difference & \\
\hline $\begin{array}{c}\text { First } \\
\text { date }\end{array}$ & $\begin{array}{c}\text { Second } \\
\text { date }\end{array}$ & $\begin{array}{c}\text { Duration } \\
\text { (days) }\end{array}$ & $\begin{array}{r}\text { First } \\
\text { date } \\
(\mathrm{cm})\end{array}$ & $\begin{array}{c}\text { Second } \\
\text { date } \\
(\mathrm{cm})\end{array}$ & $\begin{array}{c}\text { in water } \\
\text { content } \\
(\mathrm{cm})\end{array}$ & $\begin{array}{l}\text { Flux } \\
(\mathrm{cm} / \mathrm{d})\end{array}$ \\
\hline $\begin{array}{l}10-07-83 \\
10-07-83 \\
10-07-83 \\
10-07-83 \\
10-07-83 \\
10-07-83 \\
10-07-83 \\
10-07-83\end{array}$ & $\begin{array}{l}1-27-84 \\
3-07-84 \\
4-19-84 \\
5-16-84 \\
6-19-84 \\
9-04-84 \\
3-07-85 \\
6-14-85\end{array}$ & $\begin{array}{l}112 \\
152 \\
195 \\
222 \\
256 \\
333 \\
516 \\
615\end{array}$ & $\begin{array}{l}30.97 \\
30.97 \\
30.97 \\
30.97 \\
30.97 \\
30.97 \\
30.97 \\
30.97\end{array}$ & $\begin{array}{l}26.25 \\
25.98 \\
25.75 \\
25.40 \\
25.31 \\
30.46 \\
27.52 \\
24.94\end{array}$ & $\begin{array}{l}-4.73 \\
-4.99 \\
-5.22 \\
-5.58 \\
-5.66 \\
-.51 \\
-3.45 \\
-6.03\end{array}$ & $\begin{array}{r}-0.042 \\
-.033 \\
-.027 \\
-.025 \\
-.022 \\
-.002 \\
-.007 \\
-.010\end{array}$ \\
\hline $\begin{array}{l}1-27-84 \\
1-27-84 \\
1-27-84 \\
1-27-84 \\
1-27-84 \\
1-27-84 \\
1-27-84\end{array}$ & $\begin{array}{l}3-07-84 \\
4-19-84 \\
5-16-84 \\
6-19-84 \\
9-04-84 \\
3-07-85 \\
6-14-85\end{array}$ & $\begin{array}{r}40 \\
83 \\
110 \\
144 \\
220 \\
405 \\
504\end{array}$ & $\begin{array}{l}26.25 \\
26.25 \\
26.25 \\
26.25 \\
26.25 \\
26.25 \\
26.25\end{array}$ & $\begin{array}{l}25.98 \\
25.75 \\
25.40 \\
25.31 \\
30.46 \\
27.52 \\
24.94\end{array}$ & $\begin{array}{r}-.27 \\
-.50 \\
-.85 \\
-.93 \\
4.22 \\
1.27 \\
-1.31\end{array}$ & $\begin{array}{r}-.007 \\
-.006 \\
-.008 \\
-.006 \\
.019 \\
.003 \\
-.003\end{array}$ \\
\hline $\begin{array}{l}3-07-84 \\
3-07-84 \\
3-07-84 \\
3-07-84 \\
3-07-84 \\
3-07-84\end{array}$ & $\begin{array}{l}4-19-84 \\
5-16-84 \\
6-19-84 \\
9-04-84 \\
3-07-85 \\
6-14-85\end{array}$ & $\begin{array}{r}42 \\
69 \\
103 \\
180 \\
365 \\
464\end{array}$ & $\begin{array}{l}25.98 \\
25.98 \\
25.98 \\
25.98 \\
25.98 \\
25.98\end{array}$ & $\begin{array}{l}25.75 \\
25.40 \\
25.31 \\
30.46 \\
27.52 \\
24.94\end{array}$ & $\begin{array}{r}-.23 \\
-.58 \\
-.67 \\
4.48 \\
1.54 \\
-1.04\end{array}$ & $\begin{array}{r}-.005 \\
-.008 \\
-.006 \\
.025 \\
.004 \\
-.002\end{array}$ \\
\hline $\begin{array}{l}4-19-84 \\
4-19-84 \\
4-19-84 \\
4-19-84 \\
4-19-84\end{array}$ & $\begin{array}{l}5-16-84 \\
6-19-84 \\
9-04-84 \\
3-07-85 \\
6-14-85\end{array}$ & $\begin{array}{r}27 \\
61 \\
137 \\
322 \\
421\end{array}$ & $\begin{array}{l}25.75 \\
25.75 \\
25.75 \\
25.75 \\
25.75\end{array}$ & $\begin{array}{l}25.40 \\
25.31 \\
30.46 \\
27.52 \\
24.94\end{array}$ & $\begin{array}{l}-.35 \\
-.44 \\
4.71 \\
1.77 \\
-.81\end{array}$ & $\begin{array}{r}-.013 \\
-.007 \\
.034 \\
.005 \\
-.002\end{array}$ \\
\hline $\begin{array}{l}5-16-84 \\
5-16-84 \\
5-16-84 \\
5-16-84\end{array}$ & $\begin{array}{l}6-19-84 \\
9-04-84 \\
3-07-85 \\
6-14-85\end{array}$ & $\begin{array}{r}33 \\
110 \\
295 \\
394\end{array}$ & $\begin{array}{l}25.40 \\
25.40 \\
25.40 \\
25.40\end{array}$ & $\begin{array}{l}25.31 \\
30.46 \\
27.52 \\
24.94\end{array}$ & $\begin{array}{l}-.08 \\
5.07 \\
2.12 \\
-.45\end{array}$ & $\begin{array}{r}-.002 \\
.046 \\
.007 \\
-.001\end{array}$ \\
\hline $\begin{array}{l}6-19-84 \\
6-19-84 \\
6-19-84\end{array}$ & $\begin{array}{l}9-04-84 \\
3-07-85 \\
6-14-85\end{array}$ & $\begin{array}{r}76 \\
261 \\
360\end{array}$ & $\begin{array}{l}25.31 \\
25.31 \\
25.31\end{array}$ & $\begin{array}{l}30.46 \\
27.52 \\
24.94\end{array}$ & $\begin{array}{l}5.15 \\
2.21 \\
-.37\end{array}$ & $\begin{array}{r}.067 \\
.008 \\
-.001\end{array}$ \\
\hline $\begin{array}{l}9-04-84 \\
9-04-84\end{array}$ & $\begin{array}{l}3-07-85 \\
6-14-85\end{array}$ & $\begin{array}{l}184 \\
283\end{array}$ & $\begin{array}{l}30.46 \\
30.46\end{array}$ & $\begin{array}{l}27.52 \\
24.94\end{array}$ & $\begin{array}{l}-2.94 \\
-5.52\end{array}$ & $\begin{array}{l}-.016 \\
-.019\end{array}$ \\
\hline $3-07-85$ & $6-14-85$ & 99 & 27.52 & 24.94 & -2.58 & -.026 \\
\hline
\end{tabular}




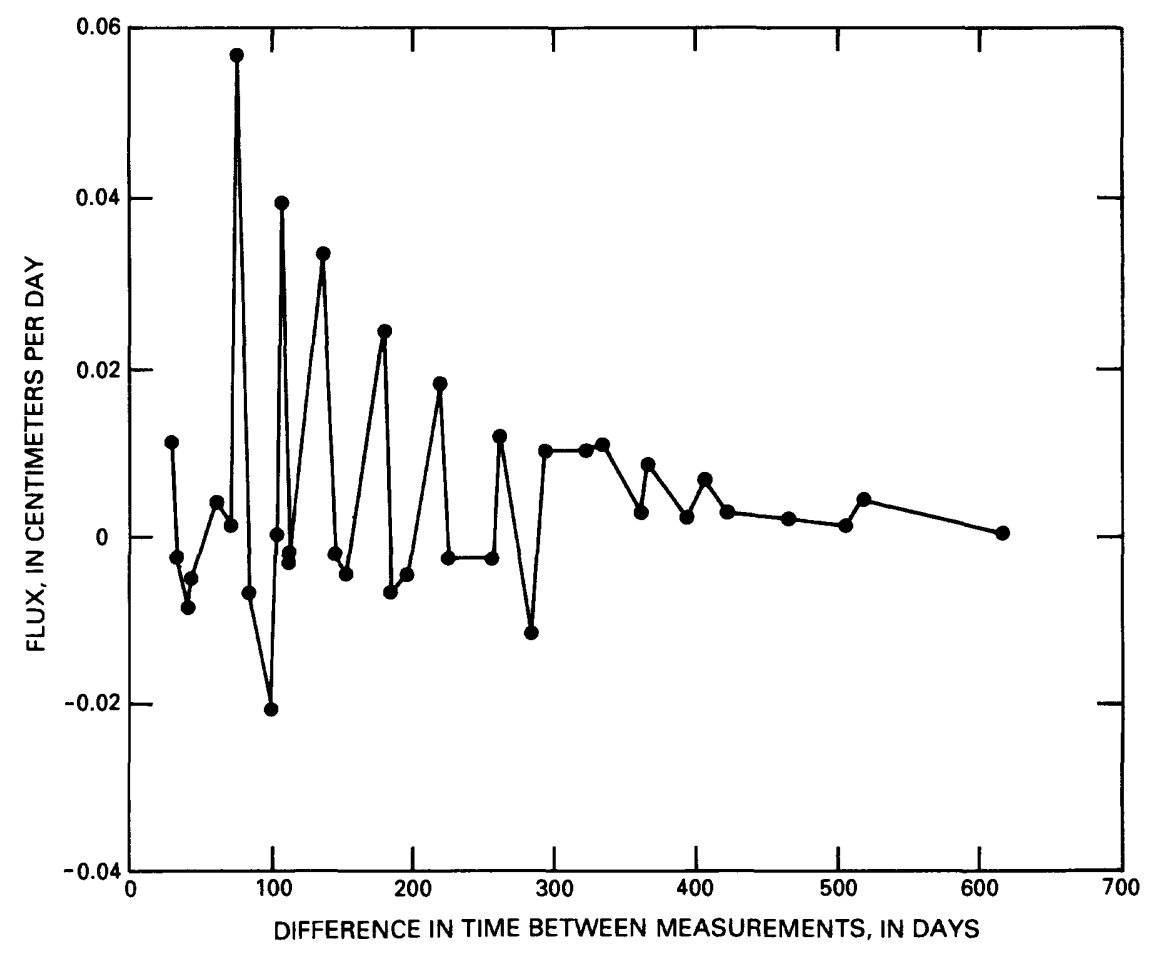

Figure 7A.--Relation between moisture flux and the difference in time between measurements for well GS-4.

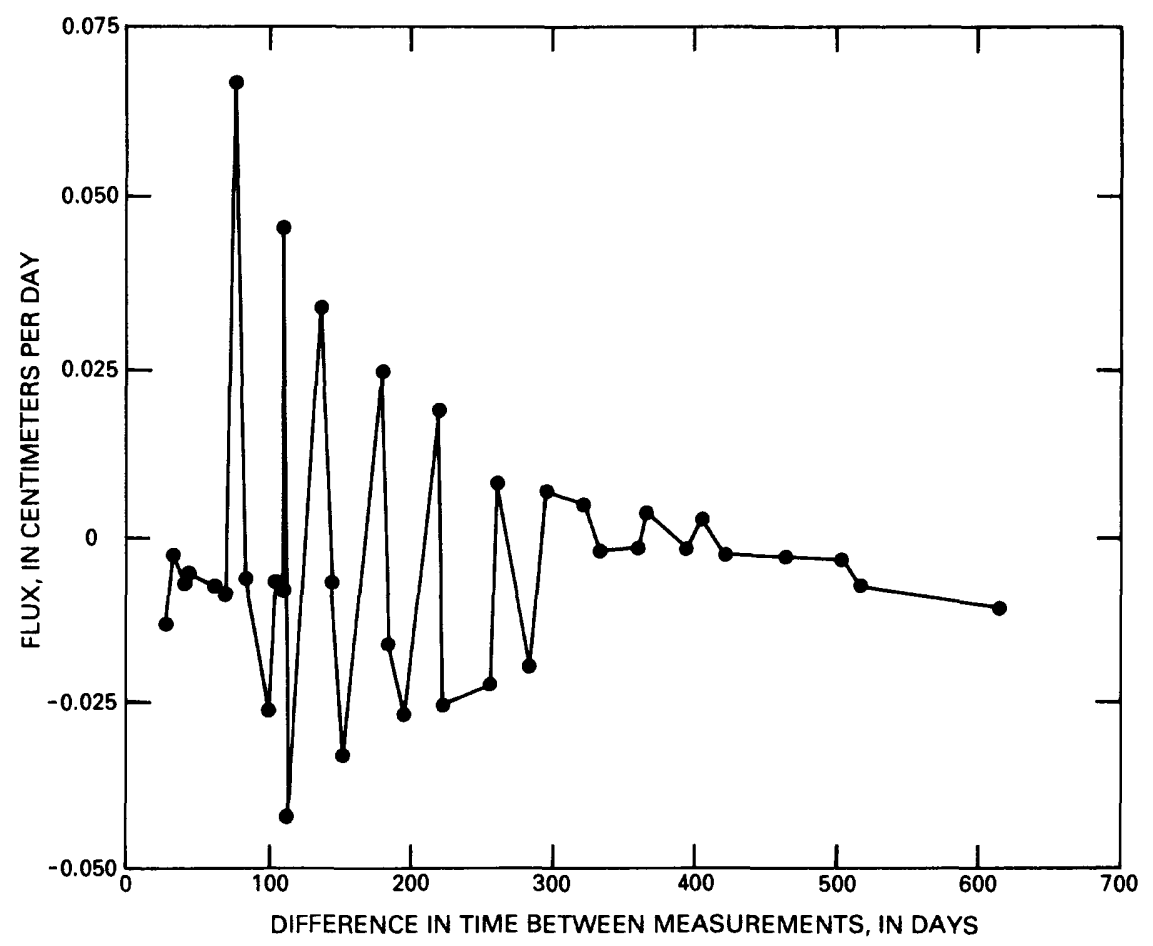

Figure 7B.--Relation between moisture flux and the difference in time between measurements for well GS-5. 
Table 4C.--Summary of flux values obtained from different neutron logs of well GS-6

[ $\mathrm{cm}$, centimeters, $\mathrm{cm} / \mathrm{d}$, centimeters per day]

\begin{tabular}{|c|c|c|c|c|c|c|}
\hline \multicolumn{2}{|c|}{ Period } & \multirow[b]{2}{*}{$\begin{array}{c}\text { Duration } \\
\text { (days) }\end{array}$} & \multicolumn{2}{|c|}{ Water content } & \multirow{2}{*}{$\begin{array}{c}\text { Difference } \\
\text { in water } \\
\text { content } \\
(\mathrm{cm})\end{array}$} & \multirow[b]{2}{*}{$\begin{array}{c}\text { Flux } \\
(\mathrm{cm} / \mathrm{d})\end{array}$} \\
\hline $\begin{array}{l}\text { First } \\
\text { date }\end{array}$ & $\begin{array}{l}\text { Second } \\
\text { date }\end{array}$ & & $\begin{array}{r}\text { First } \\
\text { date } \\
(\mathrm{cm})\end{array}$ & $\begin{array}{l}\text { Second } \\
\text { date } \\
(\mathrm{cm})\end{array}$ & & \\
\hline $\begin{array}{l}10-07-83 \\
10-07-83 \\
10-07-83 \\
10-07-83 \\
10-07-83 \\
10-07-83 \\
10-07-83 \\
10-07-83\end{array}$ & $\begin{array}{l}1-27-84 \\
3-07-84 \\
4-19-84 \\
5-16-84 \\
6-19-84 \\
9-04-84 \\
3-07-85 \\
6-14-85\end{array}$ & $\begin{array}{l}112 \\
152 \\
195 \\
222 \\
256 \\
333 \\
516 \\
615\end{array}$ & $\begin{array}{l}28.04 \\
28.04 \\
28.04 \\
28.04 \\
28.04 \\
28.04 \\
28.04 \\
28.04\end{array}$ & $\begin{array}{l}26.85 \\
25.32 \\
26.04 \\
26.46 \\
26.57 \\
31.69 \\
32.03 \\
27.73\end{array}$ & $\begin{array}{l}-1.19 \\
-2.73 \\
-2.00 \\
-1.59 \\
-1.48 \\
3.65 \\
3.99 \\
-.32\end{array}$ & $\begin{array}{r}-0.011 \\
-.018 \\
-.010 \\
-.007 \\
-.006 \\
.011 \\
.008 \\
-.001\end{array}$ \\
\hline $\begin{array}{l}1-27-84 \\
1-27-84 \\
1-27-84 \\
1-27-84 \\
1-27-84 \\
1-27-84 \\
1-27-84\end{array}$ & $\begin{array}{l}3-07-84 \\
4-19-84 \\
5-16-84 \\
6-19-84 \\
9-04-84 \\
3-07-85 \\
6-14-85\end{array}$ & $\begin{array}{r}40 \\
83 \\
110 \\
144 \\
220 \\
405 \\
504\end{array}$ & $\begin{array}{l}26.85 \\
26.85 \\
26.85 \\
26.85 \\
26.85 \\
26.85 \\
26.85\end{array}$ & $\begin{array}{l}25.32 \\
26.04 \\
26.46 \\
26.57 \\
31.69 \\
32.03 \\
27.73\end{array}$ & $\begin{array}{r}-1.53 \\
-.81 \\
-.39 \\
-.28 \\
4.84 \\
5.18 \\
.88\end{array}$ & $\begin{array}{r}-.038 \\
-.010 \\
-.004 \\
-.002 \\
.022 \\
.013 \\
.002\end{array}$ \\
\hline $\begin{array}{l}3-07-84 \\
3-07-84 \\
3-07-84 \\
3-07-84 \\
3-07-84 \\
3-07-84\end{array}$ & $\begin{array}{l}4-19-84 \\
5-16-84 \\
6-19-84 \\
9-04-84 \\
3-07-85 \\
6-14-85\end{array}$ & $\begin{array}{r}42 \\
69 \\
103 \\
180 \\
365 \\
464\end{array}$ & $\begin{array}{l}25 \cdot 32 \\
25.32 \\
25 \cdot 32 \\
25.32 \\
25 \cdot 32 \\
25.32\end{array}$ & $\begin{array}{l}26.04 \\
26.46 \\
26.57 \\
31.69 \\
32.03 \\
27.73\end{array}$ & $\begin{array}{r}.72 \\
1.14 \\
1.25 \\
6.37 \\
6.72 \\
2.41\end{array}$ & $\begin{array}{l}.017 \\
.016 \\
.012 \\
.035 \\
.018 \\
.005\end{array}$ \\
\hline $\begin{array}{l}4-19-84 \\
4-19-84 \\
4-19-84 \\
4-19-84 \\
4-19-84\end{array}$ & $\begin{array}{l}5-16-84 \\
6-19-84 \\
9-04-84 \\
3-07-85 \\
6-14-85\end{array}$ & $\begin{array}{r}27 \\
61 \\
137 \\
322 \\
421\end{array}$ & $\begin{array}{l}26.04 \\
26.04 \\
26.04 \\
26.04 \\
26.04\end{array}$ & $\begin{array}{l}26.46 \\
26.57 \\
31.69 \\
32.03 \\
27.73\end{array}$ & $\begin{array}{r}.42 \\
.53 \\
5.65 \\
5.99 \\
1.69\end{array}$ & $\begin{array}{l}.016 \\
.009 \\
.041 \\
.019 \\
.004\end{array}$ \\
\hline $\begin{array}{l}5-16-84 \\
5-16-84 \\
5-16-84 \\
5-16-84\end{array}$ & $\begin{array}{l}6-19-84 \\
9-04-84 \\
3-07-85 \\
6-14-85\end{array}$ & $\begin{array}{r}33 \\
110 \\
295 \\
394\end{array}$ & $\begin{array}{l}26.46 \\
26.46 \\
26.46 \\
26.46\end{array}$ & $\begin{array}{l}26.57 \\
31.69 \\
32.03 \\
27.73\end{array}$ & $\begin{array}{l}.11 \\
5.23 \\
5.57 \\
1.27\end{array}$ & $\begin{array}{l}.003 \\
.047 \\
.019 \\
.003\end{array}$ \\
\hline $\begin{array}{l}6-19-84 \\
6-19-84 \\
6-19-84\end{array}$ & $\begin{array}{l}9-04-84 \\
3-07-85 \\
6-14-85\end{array}$ & $\begin{array}{r}76 \\
261 \\
360\end{array}$ & $\begin{array}{l}26.57 \\
26.57 \\
26.57\end{array}$ & $\begin{array}{l}31.69 \\
32.03 \\
27.73\end{array}$ & $\begin{array}{l}5.12 \\
5.46 \\
1.16\end{array}$ & $\begin{array}{l}.067 \\
.021 \\
.003\end{array}$ \\
\hline $\begin{array}{l}9-04-84 \\
9-04-84\end{array}$ & $\begin{array}{l}3-07-85 \\
6-14-85\end{array}$ & $\begin{array}{l}184 \\
283\end{array}$ & $\begin{array}{l}31.69 \\
31.69\end{array}$ & $\begin{array}{l}32.03 \\
27.73\end{array}$ & $\begin{array}{r}.34 \\
-3.96\end{array}$ & $\begin{array}{r}.002 \\
-.014\end{array}$ \\
\hline $3-07-85$ & $6-14-85$ & 99 & 32.03 & 27.73 & -4.31 & -.043 \\
\hline
\end{tabular}


Table 4D.--Summary of flux values obtained from different neutron logs of well GS-15

[ $\mathrm{cm}$, centimeters, $\mathrm{cm} / \mathrm{d}$, centimeters per day]

\begin{tabular}{|c|c|c|c|c|c|c|}
\hline \multicolumn{2}{|c|}{ Period } & \multirow[b]{2}{*}{$\begin{array}{c}\text { Duration } \\
\text { (days) }\end{array}$} & \multicolumn{2}{|c|}{ Water content } & \multirow{2}{*}{$\begin{array}{c}\text { Difference } \\
\text { in water } \\
\text { content } \\
(\mathrm{cm})\end{array}$} & \multirow[b]{2}{*}{$\begin{array}{c}\text { Flux } \\
(\mathrm{cm} / \mathrm{d})\end{array}$} \\
\hline $\begin{array}{r}\text { First } \\
\text { date }\end{array}$ & $\begin{array}{l}\text { Second } \\
\text { date }\end{array}$ & & $\begin{array}{r}\text { First } \\
\text { date } \\
(\mathrm{cm})\end{array}$ & $\begin{array}{l}\text { Second } \\
\text { date } \\
(\mathrm{cm})\end{array}$ & & \\
\hline $\begin{array}{l}10-06-83 \\
10-06-83 \\
10-06-83 \\
10-06-83 \\
10-06-83 \\
10-06-83 \\
10-06-83 \\
10-06-83 \\
10-06-83 \\
10-06-83\end{array}$ & $\begin{array}{r}11-14-83 \\
1-27-84 \\
3-07-84 \\
4-17-84 \\
5-16-84 \\
6-19-84 \\
7-18-84 \\
9-03-84 \\
3-07-85 \\
6-14-85\end{array}$ & $\begin{array}{r}39 \\
113 \\
153 \\
194 \\
223 \\
257 \\
286 \\
333 \\
517 \\
617\end{array}$ & $\begin{array}{l}80.95 \\
80.95 \\
80.95 \\
80.95 \\
80.95 \\
80.95 \\
80.95 \\
80.95 \\
80.95 \\
80.95\end{array}$ & $\begin{array}{l}80.44 \\
78.88 \\
81.33 \\
76.98 \\
77.51 \\
77.64 \\
77.59 \\
83.54 \\
87.59 \\
89.77\end{array}$ & $\begin{array}{r}-0.51 \\
-2.07 \\
.38 \\
-3.98 \\
-3.44 \\
-3.31 \\
-3.36 \\
2.59 \\
6.64 \\
8.82\end{array}$ & $\begin{array}{r}-0.013 \\
-.018 \\
.002 \\
-.020 \\
-.015 \\
-.013 \\
-.012 \\
.008 \\
.013 \\
.014\end{array}$ \\
\hline $\begin{array}{l}11-14-83 \\
11-14-83 \\
11-14-83 \\
11-14-83 \\
11-14-83 \\
11-14-83 \\
11-14-83 \\
11-14-83 \\
11-14-83\end{array}$ & $\begin{array}{l}1-27-84 \\
3-07-84 \\
4-17-84 \\
5-16-84 \\
6-19-84 \\
7-18-84 \\
9-03-84 \\
3-07-85 \\
6-14-85\end{array}$ & $\begin{array}{r}74 \\
114 \\
155 \\
184 \\
218 \\
247 \\
294 \\
478 \\
577\end{array}$ & $\begin{array}{l}80.44 \\
80.44 \\
80.44 \\
80.44 \\
80.44 \\
80.44 \\
80.44 \\
80.44 \\
80.44\end{array}$ & $\begin{array}{l}78.88 \\
81.33 \\
76.98 \\
77.51 \\
77.64 \\
77.59 \\
83.54 \\
87.59 \\
89.77\end{array}$ & $\begin{array}{r}-1.56 \\
.89 \\
-3.47 \\
-2.94 \\
-2.80 \\
-2.86 \\
3.09 \\
7.15 \\
9.33\end{array}$ & $\begin{array}{r}-.021 \\
.008 \\
-.022 \\
-.016 \\
-.013 \\
-.012 \\
.011 \\
.015 \\
.016\end{array}$ \\
\hline $\begin{array}{l}1-27-84 \\
1-27-84 \\
1-27-84 \\
1-27-84 \\
1-27-84 \\
1-27-84 \\
1-27-84 \\
1-27-84\end{array}$ & $\begin{array}{l}3-07-84 \\
4-17-84 \\
5-16-84 \\
6-19-84 \\
7-18-84 \\
9-03-84 \\
3-07-85 \\
6-14-85\end{array}$ & $\begin{array}{r}40 \\
81 \\
110 \\
144 \\
173 \\
219 \\
405 \\
504\end{array}$ & $\begin{array}{l}78.88 \\
78.88 \\
78.88 \\
78.88 \\
78.88 \\
78.88 \\
78.88 \\
78.88\end{array}$ & $\begin{array}{l}81.33 \\
76.98 \\
77.51 \\
77.64 \\
77.59 \\
83.54 \\
87.59 \\
89.77\end{array}$ & $\begin{array}{r}2.45 \\
-1.90 \\
-1.37 \\
-1.24 \\
-1.29 \\
4.66 \\
8.71 \\
10.89\end{array}$ & $\begin{array}{r}.061 \\
-.024 \\
-.012 \\
-.009 \\
-.007 \\
.021 \\
.022 \\
.022\end{array}$ \\
\hline $\begin{array}{l}3-07-84 \\
3-07-84 \\
3-07-84 \\
3-07-84 \\
3-07-84 \\
3-07-84 \\
3-07-84\end{array}$ & $\begin{array}{l}4-17-84 \\
5-16-84 \\
6-19-84 \\
7-18-84 \\
9-03-84 \\
3-07-85 \\
6-14-85\end{array}$ & $\begin{array}{r}40 \\
69 \\
103 \\
133 \\
179 \\
365 \\
464\end{array}$ & $\begin{array}{l}81.33 \\
81.33 \\
81.33 \\
81.33 \\
81.33 \\
81.33 \\
81.33\end{array}$ & $\begin{array}{l}76.98 \\
77.51 \\
77.64 \\
77.59 \\
83.54 \\
87.59 \\
89.77\end{array}$ & $\begin{array}{r}-4.36 \\
-3.83 \\
-3.69 \\
-3.74 \\
2.21 \\
6.26 \\
8.44\end{array}$ & $\begin{array}{r}-.106 \\
-.055 \\
-.036 \\
-.028 \\
.012 \\
.017 \\
.018\end{array}$ \\
\hline $\begin{array}{l}4-17-84 \\
4-17-84 \\
4-17-84 \\
4-17-84 \\
4-17-84 \\
4-17-84\end{array}$ & $\begin{array}{l}5-16-84 \\
6-19-84 \\
7-18-84 \\
9-03-84 \\
3-07-85 \\
6-14-85\end{array}$ & $\begin{array}{r}29 \\
63 \\
92 \\
138 \\
324 \\
423\end{array}$ & $\begin{array}{l}76.98 \\
76.98 \\
76.98 \\
76.98 \\
76.98 \\
76.98\end{array}$ & $\begin{array}{l}77.51 \\
77.64 \\
77.59 \\
83.54 \\
87.59 \\
89.77\end{array}$ & $\begin{array}{r}.53 \\
.66 \\
.61 \\
6.56 \\
10.61 \\
12.79\end{array}$ & $\begin{array}{l}.018 \\
.011 \\
.007 \\
.047 \\
.033 \\
.030\end{array}$ \\
\hline
\end{tabular}


Table 4D.--Summary of flux values obtained from different neutron logs of well GS-15--Continued

\begin{tabular}{ccccccr}
\hline \multicolumn{2}{c}{ Period } & Duration & \multicolumn{2}{c}{ Water } & content \\
(days) & $\begin{array}{c}\text { First } \\
\text { date } \\
\text { date }\end{array}$ & $\begin{array}{c}\text { Second } \\
\text { date }\end{array}$ & $\begin{array}{c}\text { Second } \\
\text { date } \\
\text { (cm) }\end{array}$ & $\begin{array}{c}\text { Difference } \\
\text { in water } \\
\text { content } \\
(\mathrm{cm})\end{array}$ & $\begin{array}{c}\text { F1ux } \\
(\mathrm{cm} / \mathrm{d})\end{array}$ \\
\hline $5-16-84$ & $6-19-84$ & 33 & 77.51 & 77.64 & 0.13 & 0.004 \\
$5-16-84$ & $7-18-84$ & 63 & 77.51 & 77.59 & .08 & .001 \\
$5-16-84$ & $9-3-84$ & 109 & 77.51 & 83.54 & 6.03 & .055 \\
$5-16-84$ & $3-7-85$ & 295 & 77.51 & 87.59 & 10.08 & .034 \\
$5-16-84$ & $6-14-85$ & 394 & 77.51 & 89.77 & 12.26 & .031 \\
$6-19-84$ & $7-18-84$ & 29 & 77.64 & 77.59 & -.05 & -.002 \\
$6-19-84$ & $9-03-84$ & 75 & 77.64 & 83.54 & 5.90 & .078 \\
$6-19-84$ & $3-07-85$ & 261 & 77.64 & 87.59 & 9.95 & .038 \\
$6-19-84$ & $6-14-85$ & 360 & 77.64 & 89.77 & 12.13 & .034 \\
$7-18-84$ & $9-03-84$ & 46 & 77.59 & 83.54 & 5.95 & .127 \\
$7-18-84$ & $3-07-85$ & 232 & 77.59 & 87.59 & 10.00 & .043 \\
$7-18-84$ & $6-14-85$ & 331 & 77.59 & 89.77 & 12.18 & .037 \\
$9-03-84$ & $3-07-85$ & 185 & 83.54 & 87.59 & 4.05 & .022 \\
$9-03-84$ & $6-14-85$ & 284 & 83.54 & 89.77 & 6.23 & .022 \\
$3-07-85$ & $6-14-85$ & 99 & 87.59 & 89.77 & 2.18 & .022 \\
\hline
\end{tabular}




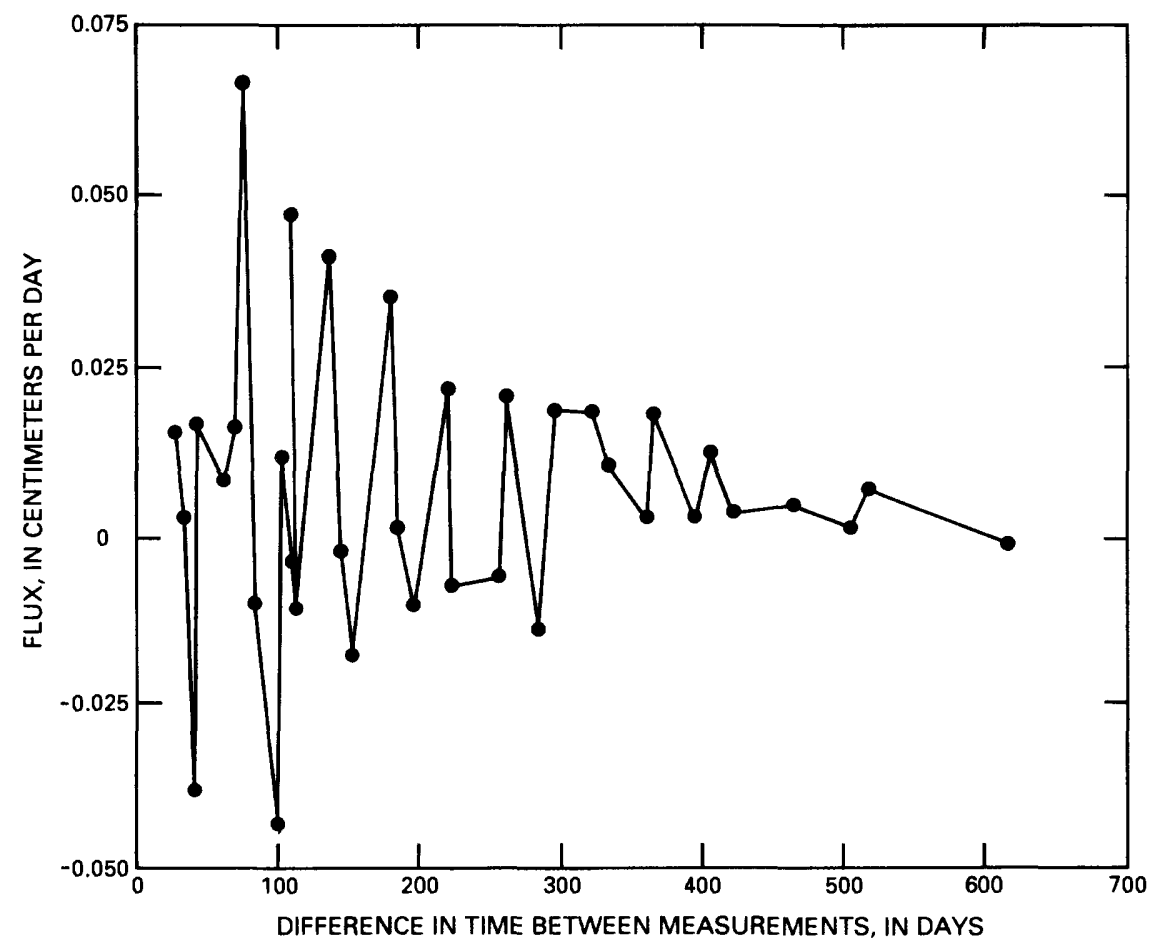

Figure 7C.--Relation between moisture flux and the difference in time between measurements for well GS- 6 .

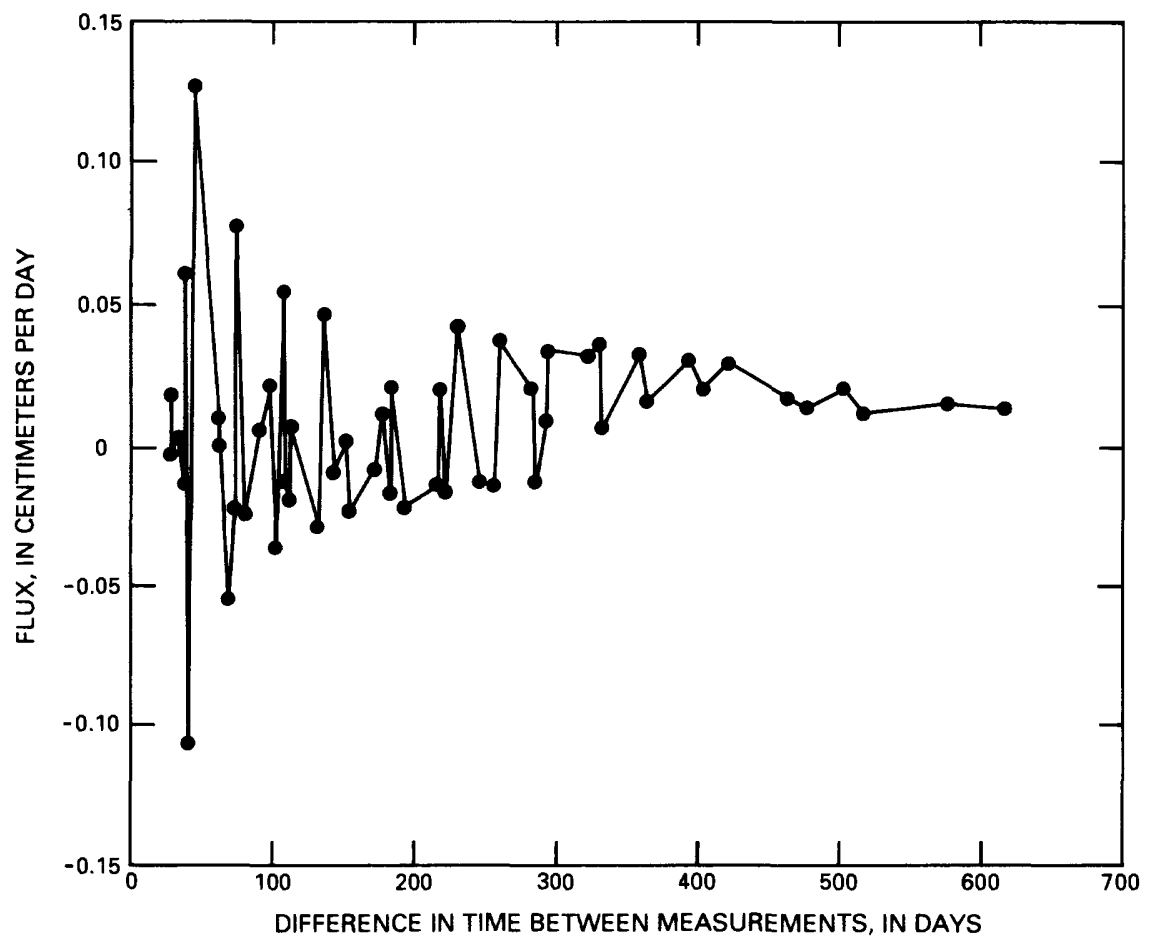

Figure $7 D--$ Relation between moisture flux and the difference in time between measurements for well GS-15. 
Table 4E.--Summary of flux values obtained from different neutron logs of well GS-18

[ $\mathrm{cm}$, centimeters, $\mathrm{cm} / \mathrm{d}$, centimeters per day]

\begin{tabular}{|c|c|c|c|c|c|c|}
\hline \multicolumn{2}{|c|}{ Period } & \multirow[b]{2}{*}{$\begin{array}{l}\text { Duration } \\
\text { (days) }\end{array}$} & \multicolumn{2}{|c|}{ Water content } & \multirow{2}{*}{$\begin{array}{c}\text { Difference } \\
\text { in water } \\
\text { content } \\
(\mathrm{cm})\end{array}$} & \multirow[b]{2}{*}{$\underset{(\mathrm{cm} / \mathrm{d})}{\text { Flux }}$} \\
\hline $\begin{array}{c}\text { First } \\
\text { date }\end{array}$ & $\begin{array}{l}\text { Second } \\
\text { date }\end{array}$ & & $\begin{array}{r}\text { First } \\
\text { date } \\
(\mathrm{cm})\end{array}$ & $\begin{array}{l}\text { Second } \\
\text { date } \\
(\mathrm{cm})\end{array}$ & & \\
\hline $\begin{array}{l}10-06-83 \\
10-06-83 \\
10-06-83 \\
10-06-83 \\
10-06-83 \\
10-06-83 \\
10-06-83 \\
10-06-83\end{array}$ & $\begin{array}{l}1-27-84 \\
3-07-84 \\
5-17-84 \\
6-19-84 \\
7-18-84 \\
9-03-84 \\
3-08-85 \\
6-15-85\end{array}$ & $\begin{array}{l}113 \\
153 \\
224 \\
257 \\
286 \\
333 \\
519 \\
617\end{array}$ & $\begin{array}{l}76.09 \\
76.09 \\
76.09 \\
76.09 \\
76.09 \\
76.09 \\
76.09 \\
76.09\end{array}$ & $\begin{array}{l}69.64 \\
68.11 \\
68.73 \\
69.00 \\
68.94 \\
85.77 \\
79.68 \\
78.58\end{array}$ & $\begin{array}{r}-6.44 \\
-7.98 \\
-7.36 \\
-7.09 \\
-7.14 \\
9.69 \\
3.60 \\
2.49\end{array}$ & $\begin{array}{r}-0.057 \\
-.052 \\
-.033 \\
-.028 \\
-.025 \\
.029 \\
.007 \\
.004\end{array}$ \\
\hline $\begin{array}{l}1-27-84 \\
1-27-84 \\
1-27-84 \\
1-27-84 \\
1-27-84 \\
1-27-84 \\
1-27-84\end{array}$ & $\begin{array}{l}3-07-84 \\
5-17-84 \\
6-19-84 \\
7-18-84 \\
9-03-84 \\
3-08-85 \\
6-15-85\end{array}$ & $\begin{array}{r}40 \\
111 \\
144 \\
173 \\
219 \\
406 \\
505\end{array}$ & $\begin{array}{l}69.64 \\
69.64 \\
69.64 \\
69.64 \\
69.64 \\
69.64 \\
69.64\end{array}$ & $\begin{array}{l}68.11 \\
68.73 \\
69.00 \\
68.94 \\
85.77 \\
79.68 \\
78.58\end{array}$ & $\begin{array}{r}-1.53 \\
-.91 \\
-.65 \\
-.70 \\
16.13 \\
10.04 \\
8.93\end{array}$ & $\begin{array}{l}-.038 \\
-.008 \\
-.004 \\
-.004 \\
.073 \\
.025 \\
.018\end{array}$ \\
\hline $\begin{array}{l}3-07-84 \\
3-07-84 \\
3-07-84 \\
3-07-84 \\
3-07-84 \\
3-07-84\end{array}$ & $\begin{array}{l}5-17-84 \\
6-19-84 \\
7-18-84 \\
9-03-84 \\
3-08-85 \\
6-15-85\end{array}$ & $\begin{array}{r}70 \\
103 \\
133 \\
179 \\
366 \\
465\end{array}$ & $\begin{array}{l}68.11 \\
68.11 \\
68.11 \\
68.11 \\
68.11 \\
68.11\end{array}$ & $\begin{array}{l}68.73 \\
69.00 \\
68.94 \\
85.77 \\
79.68 \\
78.58\end{array}$ & $\begin{array}{r}.62 \\
.89 \\
.83 \\
17.66 \\
11.57 \\
10.47\end{array}$ & $\begin{array}{l}.009 \\
.009 \\
.006 \\
.098 \\
.032 \\
.023\end{array}$ \\
\hline $\begin{array}{l}5-17-84 \\
5-17-84 \\
5-17-84 \\
5-17-84 \\
5-17-84\end{array}$ & $\begin{array}{l}6-19-84 \\
7-18-84 \\
9-03-84 \\
3-08-85 \\
6-15-85\end{array}$ & $\begin{array}{r}33 \\
62 \\
108 \\
295 \\
394\end{array}$ & $\begin{array}{l}68.73 \\
68.73 \\
68.73 \\
68.73 \\
68.73\end{array}$ & $\begin{array}{l}69.00 \\
68.94 \\
85.77 \\
79.68 \\
78.58\end{array}$ & $\begin{array}{r}.27 \\
.22 \\
17.04 \\
10.95 \\
9.85\end{array}$ & $\begin{array}{l}.008 \\
.003 \\
.156 \\
.037 \\
.025\end{array}$ \\
\hline $\begin{array}{l}6-19-84 \\
6-19-84 \\
6-19-84 \\
6-19-84\end{array}$ & $\begin{array}{l}7-18-84 \\
9-03-84 \\
3-08-85 \\
6-15-85\end{array}$ & $\begin{array}{r}29 \\
75 \\
262 \\
361\end{array}$ & $\begin{array}{l}69.00 \\
69.00 \\
69.00 \\
69.00\end{array}$ & $\begin{array}{l}68.94 \\
85.77 \\
79.68 \\
78.58\end{array}$ & $\begin{array}{r}-.05 \\
16.78 \\
10.69 \\
9.58\end{array}$ & $\begin{array}{r}-.002 \\
.221 \\
.041 \\
.027\end{array}$ \\
\hline $\begin{array}{l}7-18-84 \\
7-18-84 \\
7-18-84\end{array}$ & $\begin{array}{l}9-03-84 \\
3-08-85 \\
6-15-85\end{array}$ & $\begin{array}{r}46 \\
233 \\
332\end{array}$ & $\begin{array}{l}68.94 \\
68.94 \\
68.94\end{array}$ & $\begin{array}{l}85.77 \\
79.68 \\
78.58\end{array}$ & $\begin{array}{r}16.83 \\
10.74 \\
9.63\end{array}$ & $\begin{array}{l}.358 \\
.046 \\
.029\end{array}$ \\
\hline $\begin{array}{l}9-03-84 \\
9-03-84\end{array}$ & $\begin{array}{l}3-08-85 \\
6-15-85\end{array}$ & $\begin{array}{l}186 \\
285\end{array}$ & $\begin{array}{l}85.77 \\
85.77\end{array}$ & $\begin{array}{l}79.68 \\
78.58\end{array}$ & $\begin{array}{l}-6.09 \\
-7.19\end{array}$ & $\begin{array}{l}-.033 \\
-.025\end{array}$ \\
\hline $3-08-85$ & $6-15-85$ & 98 & 79.68 & 78.58 & -1.10 & -.011 \\
\hline
\end{tabular}


Table 4F.--Summary of flux values obtained from different neutron logs of well GS-20

[ $\mathrm{cm}$, centimeters, $\mathrm{cm} / \mathrm{d}$, centimeters per day]

\begin{tabular}{|c|c|c|c|c|c|c|}
\hline \multicolumn{2}{|c|}{ Period } & \multirow[b]{2}{*}{$\begin{array}{c}\text { Duration } \\
\text { (days) }\end{array}$} & \multicolumn{2}{|c|}{ Water content } & \multirow{2}{*}{$\begin{array}{c}\text { Difference } \\
\text { in water } \\
\text { content } \\
(\mathrm{cm})\end{array}$} & \multirow[b]{2}{*}{$\underset{(\mathrm{cm} / \mathrm{d})}{\mathrm{Flux}}$} \\
\hline $\begin{array}{c}\text { First } \\
\text { date }\end{array}$ & $\begin{array}{c}\text { Second } \\
\text { date }\end{array}$ & & $\begin{array}{c}\text { First } \\
\text { date } \\
(\mathrm{cm})\end{array}$ & $\begin{array}{c}\text { Second } \\
\text { date } \\
(\mathrm{cm})\end{array}$ & & \\
\hline $\begin{array}{l}10-07-83 \\
10-07-83 \\
10-07-83 \\
10-07-83 \\
10-07-83 \\
10-07-83 \\
10-07-83 \\
10-07-83 \\
10-07-83 \\
10-07-83\end{array}$ & $\begin{array}{r}11-11-83 \\
1-28-84 \\
3-07-84 \\
4-18-84 \\
5-17-84 \\
6-18-84 \\
7-17-84 \\
9-01-84 \\
3-07-85 \\
6-14-85\end{array}$ & $\begin{array}{r}34 \\
113 \\
152 \\
194 \\
223 \\
255 \\
284 \\
330 \\
516 \\
615\end{array}$ & $\begin{array}{l}123.57 \\
123.57 \\
123.57 \\
123.57 \\
123.57 \\
123.57 \\
123.57 \\
123.57 \\
123.57 \\
123.57\end{array}$ & $\begin{array}{l}123.55 \\
123.41 \\
123.04 \\
121.97 \\
122.61 \\
122.30 \\
121.81 \\
127.05 \\
128.23 \\
123.92\end{array}$ & $\begin{array}{r}-0.02 \\
-.15 \\
-.53 \\
-1.59 \\
-.96 \\
-1.27 \\
-1.76 \\
3.48 \\
4.66 \\
.35\end{array}$ & $\begin{array}{r}-0.001 \\
-.001 \\
-.003 \\
-.008 \\
-.004 \\
-.005 \\
-.006 \\
.011 \\
.009 \\
.001\end{array}$ \\
\hline $\begin{array}{l}11-11-83 \\
11-11-83 \\
11-11-83 \\
11-11-83 \\
11-11-83 \\
11-11-83 \\
11-11-83 \\
11-11-83 \\
11-11-83\end{array}$ & $\begin{array}{l}1-28-84 \\
3-07-84 \\
4-18-84 \\
5-17-84 \\
6-18-84 \\
7-17-84 \\
9-01-84 \\
3-07-85 \\
6-14-85\end{array}$ & $\begin{array}{r}78 \\
117 \\
159 \\
188 \\
220 \\
249 \\
295 \\
481 \\
581\end{array}$ & $\begin{array}{l}123.55 \\
123.55 \\
123.55 \\
123.55 \\
123.55 \\
123.55 \\
123.55 \\
123.55 \\
123.55\end{array}$ & $\begin{array}{l}123.41 \\
123.04 \\
121.97 \\
122.61 \\
122.30 \\
121.81 \\
127.05 \\
128.23 \\
123.92\end{array}$ & $\begin{array}{r}-.13 \\
-.50 \\
-1.57 \\
-.93 \\
-1.25 \\
-1.73 \\
3.51 \\
4.68 \\
.38\end{array}$ & $\begin{array}{r}-.002 \\
-.004 \\
-.010 \\
-.005 \\
-.006 \\
-.007 \\
.012 \\
.010 \\
.001\end{array}$ \\
\hline $\begin{array}{l}1-28-84 \\
1-28-84 \\
1-28-84 \\
1-28-84 \\
1-28-84 \\
1-28-84 \\
1-28-84 \\
1-28-84\end{array}$ & $\begin{array}{l}3-07-84 \\
4-18-84 \\
5-17-84 \\
6-18-84 \\
7-17-84 \\
9-01-84 \\
3-07-85 \\
6-14-85\end{array}$ & $\begin{array}{r}39 \\
81 \\
110 \\
142 \\
170 \\
216 \\
404 \\
503\end{array}$ & $\begin{array}{l}123.41 \\
123.41 \\
123.41 \\
123.41 \\
123.41 \\
123.41 \\
123.41 \\
123.41\end{array}$ & $\begin{array}{l}123.04 \\
121.97 \\
122.61 \\
122.30 \\
121.81 \\
127.05 \\
128.23 \\
123.92\end{array}$ & $\begin{array}{r}-.37 \\
-1.44 \\
-.80 \\
-1.12 \\
-1.60 \\
3.64 \\
4.81 \\
.51\end{array}$ & $\begin{array}{r}-.009 \\
-.018 \\
-.007 \\
-.008 \\
-.009 \\
.017 \\
.012 \\
.001\end{array}$ \\
\hline $\begin{array}{l}3-07-84 \\
3-07-84 \\
3-07-84 \\
3-07-84 \\
3-07-84 \\
3-07-84 \\
3-07-84\end{array}$ & $\begin{array}{l}4-18-84 \\
5-17-84 \\
6-18-84 \\
7-17-84 \\
9-01-84 \\
3-07-85 \\
6-14-85\end{array}$ & $\begin{array}{r}41 \\
70 \\
102 \\
131 \\
177 \\
365 \\
464\end{array}$ & $\begin{array}{l}123.04 \\
123.04 \\
123.04 \\
123.04 \\
123.04 \\
123.04 \\
123.04\end{array}$ & $\begin{array}{l}121.97 \\
122.61 \\
122.30 \\
121.81 \\
127.05 \\
128.23 \\
123.92\end{array}$ & $\begin{array}{r}-1.07 \\
-.43 \\
-.75 \\
-1.23 \\
4.01 \\
5.18 \\
.88\end{array}$ & $\begin{array}{r}-.025 \\
-.006 \\
-.007 \\
-.009 \\
.023 \\
.014 \\
.002\end{array}$ \\
\hline $\begin{array}{l}4-18-84 \\
4-18-84 \\
4-18-84 \\
4-18-84 \\
4-18-84 \\
4-18-84\end{array}$ & $\begin{array}{l}5-17-84 \\
6-18-84 \\
7-17-84 \\
9-01-84 \\
3-07-85 \\
6-14-85\end{array}$ & $\begin{array}{r}29 \\
61 \\
89 \\
135 \\
323 \\
422\end{array}$ & $\begin{array}{l}121.97 \\
121.97 \\
121.97 \\
121.97 \\
121.97 \\
121.97\end{array}$ & $\begin{array}{l}122.61 \\
122.30 \\
121.81 \\
127.05 \\
128.23 \\
123.92\end{array}$ & $\begin{array}{r}.64 \\
.32 \\
-.16 \\
5.08 \\
6.25 \\
1.95\end{array}$ & $\begin{array}{r}.022 \\
.005 \\
-.002 \\
0.037 \\
.019 \\
.005\end{array}$ \\
\hline
\end{tabular}


Table 4F.--Summary of flux values obtained from different neutron logs of well GS-20--Continued

\begin{tabular}{|c|c|c|c|c|c|c|}
\hline \multicolumn{2}{|c|}{ Period } & \multirow[b]{2}{*}{$\begin{array}{c}\text { Duration } \\
\text { (days) }\end{array}$} & \multicolumn{2}{|c|}{ Water content } & \multirow{2}{*}{$\begin{array}{c}\text { Difference } \\
\text { in water } \\
\text { content } \\
(\mathrm{cm})\end{array}$} & \multirow[b]{2}{*}{$\underset{(\mathrm{cm} / \mathrm{d})}{\operatorname{Flux}}$} \\
\hline $\begin{array}{c}\text { First } \\
\text { date }\end{array}$ & $\begin{array}{l}\text { Second } \\
\text { date }\end{array}$ & & $\begin{array}{r}\text { First } \\
\text { date } \\
(\mathrm{cm})\end{array}$ & $\begin{array}{c}\text { Second } \\
\text { date } \\
(\mathrm{cm})\end{array}$ & & \\
\hline $\begin{array}{l}5-17-84 \\
5-17-84 \\
5-17-84 \\
5-17-84 \\
5-17-84\end{array}$ & $\begin{array}{l}6-18-84 \\
7-17-84 \\
9-01-84 \\
3-07-85 \\
6-14-85\end{array}$ & $\begin{array}{r}32 \\
60 \\
106 \\
294 \\
393\end{array}$ & $\begin{array}{l}122.61 \\
122.61 \\
122.61 \\
122.61 \\
122.61\end{array}$ & $\begin{array}{l}122.30 \\
121.81 \\
127.05 \\
128.23 \\
123.92\end{array}$ & $\begin{array}{l}-.32 \\
-.80 \\
4.44 \\
5.61 \\
1.31\end{array}$ & $\begin{array}{r}-.010 \\
-.013 \\
.042 \\
.019 \\
.003\end{array}$ \\
\hline $\begin{array}{l}6-18-84 \\
6-18-84 \\
6-18-84 \\
6-18-84\end{array}$ & $\begin{array}{l}7-17-84 \\
9-01-84 \\
3-07-85 \\
6-14-85\end{array}$ & $\begin{array}{r}28 \\
74 \\
262 \\
361\end{array}$ & $\begin{array}{l}122.30 \\
122.30 \\
122.30 \\
122.30\end{array}$ & $\begin{array}{l}121.81 \\
127.05 \\
128.23 \\
123.92\end{array}$ & $\begin{array}{l}-.48 \\
4.76 \\
5.93 \\
1.63\end{array}$ & $\begin{array}{r}-.017 \\
.063 \\
.023 \\
.005\end{array}$ \\
\hline $\begin{array}{l}7-17-84 \\
7-17-84 \\
7-17-84\end{array}$ & $\begin{array}{l}9-01-84 \\
3-07-85 \\
6-14-85\end{array}$ & $\begin{array}{r}46 \\
233 \\
332\end{array}$ & $\begin{array}{l}121.81 \\
121.81 \\
121.81\end{array}$ & $\begin{array}{l}127.05 \\
128.23 \\
123.92\end{array}$ & $\begin{array}{l}5.24 \\
6.41 \\
2.11\end{array}$ & $\begin{array}{l}.114 \\
.027 \\
.006\end{array}$ \\
\hline $\begin{array}{l}9-01-84 \\
9-01-84\end{array}$ & $\begin{array}{l}3-07-85 \\
6-14-85\end{array}$ & $\begin{array}{l}187 \\
286\end{array}$ & $\begin{array}{l}127.05 \\
127.05\end{array}$ & $\begin{array}{l}128.23 \\
123.92\end{array}$ & $\begin{array}{r}1.17 \\
-3.13\end{array}$ & $\begin{array}{r}.006 \\
-.011\end{array}$ \\
\hline $3-07-85$ & $6-14-85$ & 99 & 128.23 & 123.92 & -4.30 & -.043 \\
\hline
\end{tabular}




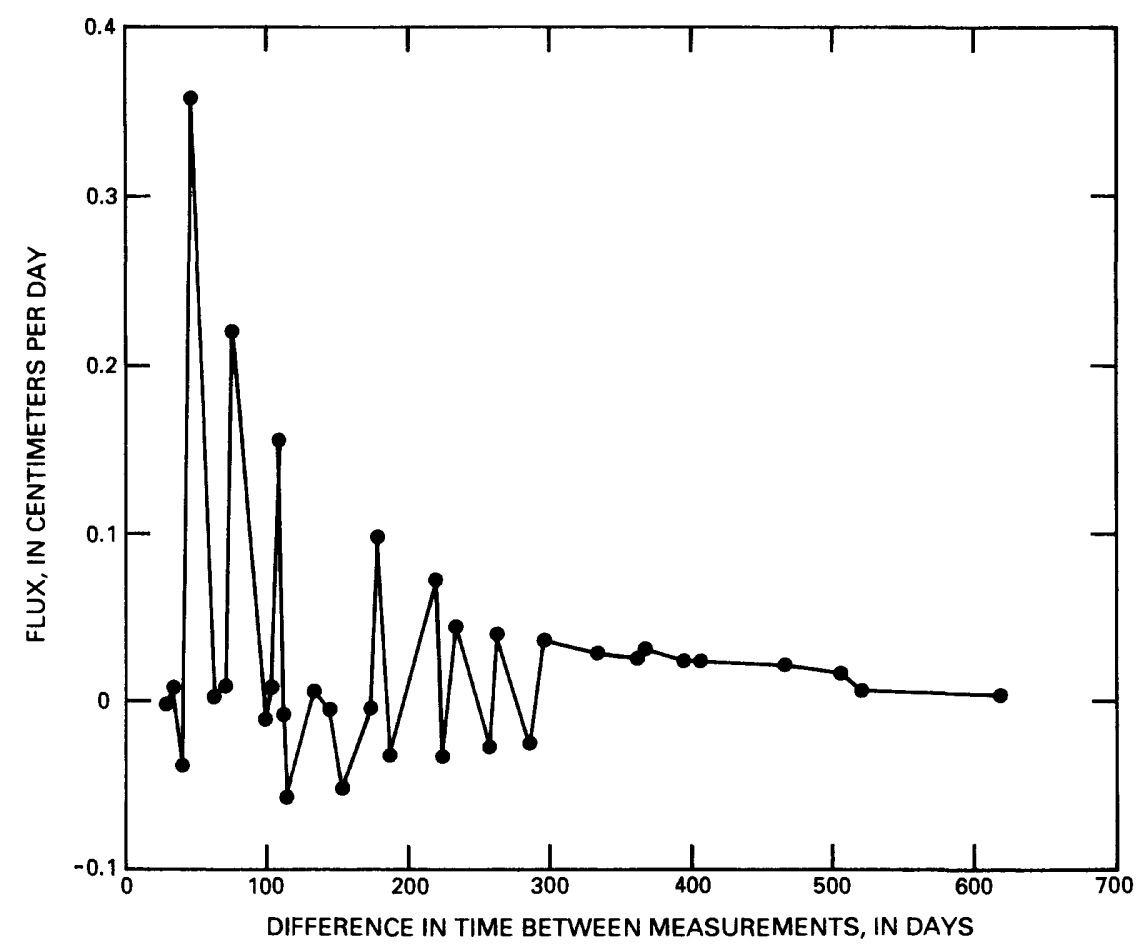

Figure $7 E .--$ Relation between moisture flux and the difference in time between measurements for well GS-18.

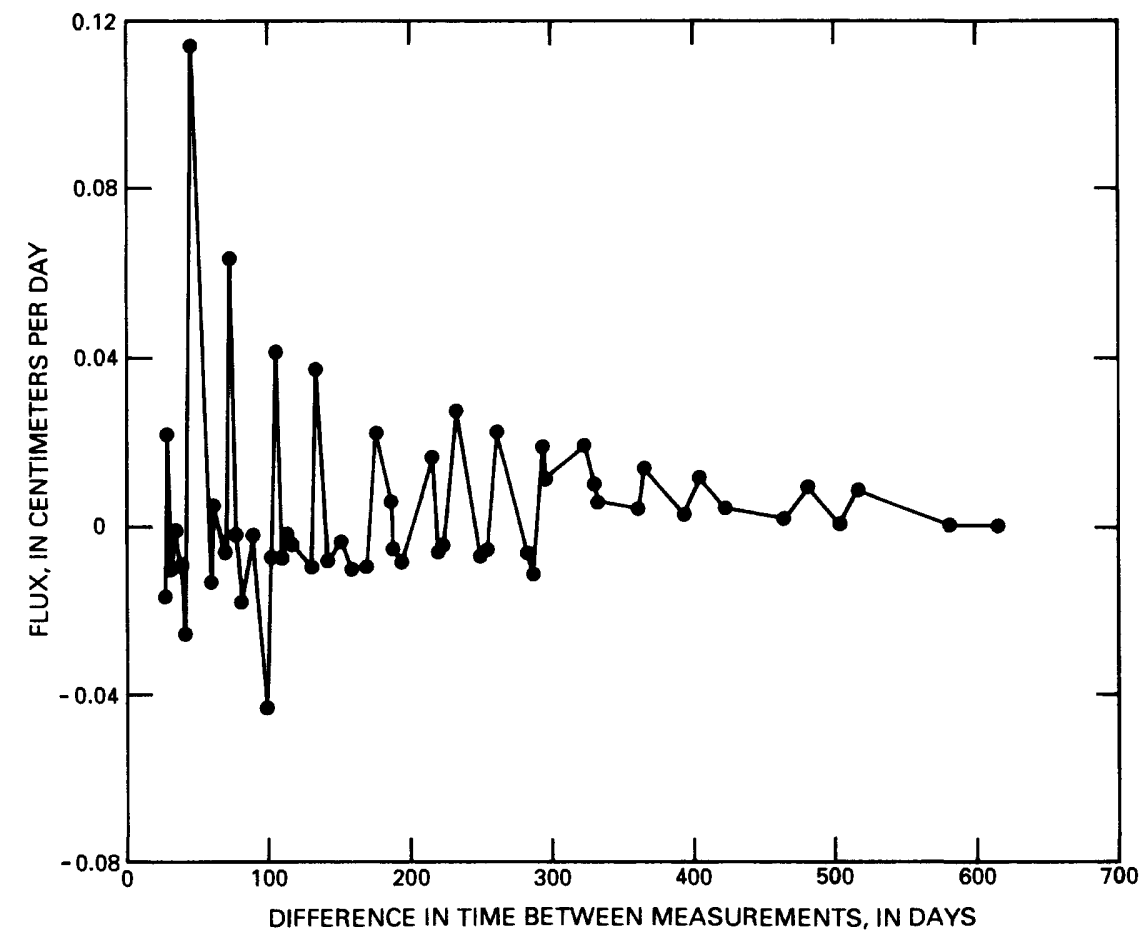

Figure 7F.--Relation between moisture flux and the difference in time between measurements for well GS-20. 


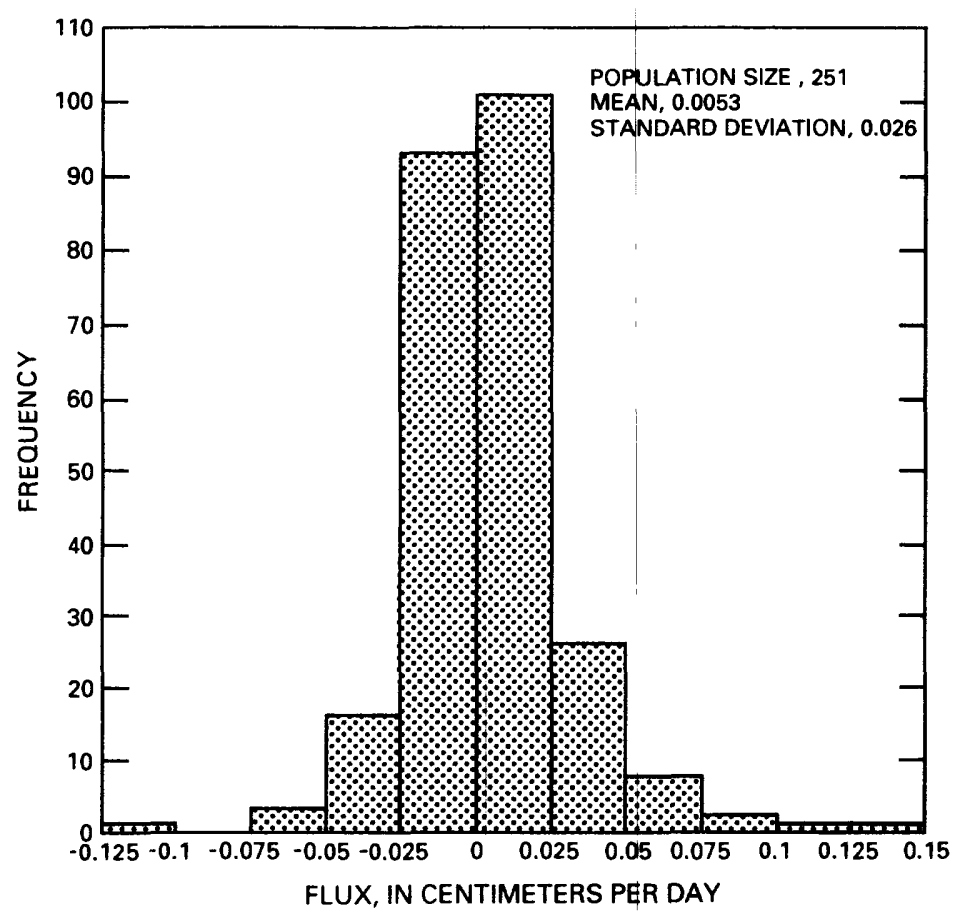

Figure 8.--Distribution of fluxes estimated from changes in soil-moisture content with time.

Figures $(7 A-F)$ show positive fluxes, indicating an increase in soil moisture with time; and negative fluxes, indicating a decrease in soil moisture with time. Although the times at which sampling occurred are sparse, all of the plots show clusters of points at about $0.0 \mathrm{~cm} / \mathrm{d}$. A strong grouping of positive fluxes at GS-20 is shown in figure $7 F$; GS-20 is located adjacent to the Amargosa River stream channel. Generally, 1983 received less precipitation than 1984 and 1985 .

A limitation to this method is that, although a flux magnitude may be estimated, direction cannot be estimated only from the water content of the soil-moisture profiles. The flow direction might be determined by comparing neutron logs side by side and recording moisture-pulse movements in the profile (fig. 5). This method also has limitations in its applicability for estimating evapotranspiration. The greatest limitation probably is that data for the upper $30 \mathrm{~cm}$ of the soil-moisture profile could not be recorded because of the design of the soil-moisture probe. The largest changes in soil moisture likely may occur in the upper $30 \mathrm{~cm}$. Increases or decreases in moisture content in the unsaturated zone can result from changes in watertable position or from recharge that results from precipitation or storm runoff. Evaporative fluxes estimated by this technique give larger magnitude values for smaller time differences and smaller magnitude values for larger times, as would be expected using time as the flux denominator. 


\section{SUMMARY}

Hydrologic and other data presented in this report were collected to further characterize the geohydrology and ground-water discharge at Franklin Lake playa, Inyo County, California. These data include: (1) Hydrographs of water levels in piezometers; (2) vertical hydraulic gradients estimated from piezometer-nest data; (3) meteorological data from weather stations in the vicinity of Franklin Lake playa; and (4) estimates of moisture fluxes based on changes in soil-moisture content in the unsaturated zone.

Hydrographs of water levels in piezometers vary from being very smooth to having extreme fluctuations. Average water-level altitudes from these hydrographs can be used to contour the water-table altitude in the vicinity of Franklin Lake playa. Vertical hydraulic gradients estimated from piezometernest data can be used to estimate ground-water discharge, provided reliable estimates of vertical hydraulic conductivity are available. Evapotranspiration estimates based on changes in soil-moisture content in the unsaturated zone provide a basis of comparison for other methods such as the energy-budget eddy-correlation technique. 
Calzia, J.P., Crowley, J.A., Dockter, R.D., Simoni, T.R., and Server, G.T., 1979, Leasable mineral resources of the California Desert Conservation Area: U.S. Geological Survey Administrative Report, 53 p., appendix II A-L.

Czarnecki, J.B., 1985, Simulated effects of increased recharge on the groundwater flow system of Yucca Mountain and vicinity, Nevada-California: U.S. Geological Survey Water-Resources Investigations Report 84-4344, $33 \mathrm{p}$.

Czarnecki, J.B., 1987, Characterization of the subregional ground-water flow system of a potential site for a high-level nuclear waste repository: Ph.D. dissertation, Univ. of Minnesota, University Microfilms, Ann Arbor, Michigan.

Czarnecki, J.B., and Waddell, R.K., 1984, Finite-element simulation of groundwater flow in the vicinity of Yucca Mountain, Nevada-California: U.S. Geological Survey Water-Resources Report 84-4349, 38 p.

Eakin, T.E., Maxey, G.B., Robinson, T.W., Fredericks, J.C., and Loeltz, 0.J., 1951, Contributions to the hydrology of eastern Nevada: Nevada Department of Conservation and Natural Resources Water Resources Bulletin 12, p. 14-16.

Pantea, M.P., 1980, Lithology and lithium content of sediments drilled in a test hole on Alkali Flat (Franklin Lake), Inyo County, California: U.S. Geological Survey Open-File Report 80-1164, 20 p.

Rush, F.E., 1970, Regional ground-water systems in the Nevada Test Site area, Nye, Lincoln, and Clark Counties, Nevada: Nevada Department of Conservation and Natural Resources Water Resources Reconnaissance Series Report 54, p. 10-16.

Waddell, R.K., 1982, Two-dimensional, steady-state model of ground-water flow, Nevada Test Site and vicinity, Nevada-California: U.S. Geological Survey Water-Resources Investigations 82-4085, $72 \mathrm{p}$.

Walker, G.E., and Eakin, T.E., 1963, Geology and groundwater of Amargosa Desert, Nevada-California: Reno, Nevada Department of Conservation and Natural Resources Ground-Water Resources-Reconnaissance Series Report 14, $45 \mathrm{p}$.

Winograd, I.J., and Thordarson, William, 1975, Hydrogeologic and hydrochemical framework, south-central Great Basin, Nevada-California, with special reference to the Nevada Test Site: U.S. Geological Survey Professional Paper 712-C, 126 p. 